\title{
GCU
}

Glasgow Caledonian

University

University for the Common Good

\section{A model predictive vertical motion control of a passenger ship}

Kucukdemiral, Ibrahim Beklan; Cakici, Ferdi; Yazici, Hakan

Published in:

Ocean Engineering

DOI:

10.1016/j.oceaneng.2019.06.005

Publication date:

2019

Document Version

Author accepted manuscript

Link to publication in ResearchOnline

Citation for published version (Harvard):

Kucukdemiral, IB, Cakici, F \& Yazici, H 2019, 'A model predictive vertical motion control of a passenger ship', Ocean Engineering, vol. 186, 106100. https://doi.org/10.1016/j.oceaneng.2019.06.005

Copyright and moral rights for the publications made accessible in the public portal are retained by the authors and/or other copyright owners and it is a condition of accessing publications that users recognise and abide by the legal requirements associated with these rights.

Take down policy

If you believe that this document breaches copyright please view our takedown policy at https://edshare.gcu.ac.uk/id/eprint/5179 for details of how to contact us. 


\author{
A Model Predictive Vertical Motion Control of a Passenger Ship \\ Ibrahim Beklan Kucukdemiral ${ }^{1}$ \\ Glasgow Caledonian University, School of Computing, Engineering and Built Environment, Department of Applied Science, \\ Glasgow, UK \\ Ferdi Cakici ${ }^{2}$ \\ Yildiz Technical University, Department of Naval Architecture and Marine Engineering, Istanbul, Turkey \\ Hakan Yazici ${ }^{3}$ \\ Yildiz Technical University, Department of Mechanical Engineering, Istanbul, Turkey
}

\begin{abstract}
In this study, the design problem of a Model Predictive Controller (MPC) for attenuation of vertical motions of a passenger ship which is subject to irregular wave excitations is investigated. The proposed design considers actuator amplitude and rate saturation phenomenon. The motion control system of the ship utilises a pair of active stabilizing fins mounted to the head and tail. First, irregular long crested head waves are implemented by a well-established randomization theory in order to find heave force and pitch moment at $F_{n}=0.40$ and $F_{n}=0.50$ in the time domain. Then, a two-degree-of-freedom mathematical model, in which pitch and heave motions are coupled with the approximation of convolution integrals is solved to obtain the uncontrolled motions and accelerations of the ship. Finally, considering the physical amplitude and rate limitations of the active fin mechanism, an MPC design is proposed to obtain a practically applicable state-feedback control law for attenuating vertical motion of a passenger ship. The performance of the MPC is also compared with an elipsoid based $\mathcal{H}_{\infty}$ controller. An extensive amount of simulation studies are presented at the end to illustrate the effectiveness of the proposed approach.
\end{abstract}

Keywords: Model Predictive Control, $\mathcal{H}_{\infty}$ Control, Magnitude and Rate Saturated Actuators, Vertical Motion of Ship, Irregular Sea Waves, Seasickness

2010 MSC: 00-01, 99-00

\footnotetext{
*Corresponding author

Email address: hyazici@yildiz.edu.tr (Hakan Yazici)

${ }^{1}$ Glasgow G4 0BA

${ }^{2} 34349$ Yildiz-Istanbul

${ }^{3} 34349$ Yildiz-Istanbul
} 


\section{Introduction}

Seakeeping ability of a ship is strongly associated with the improvements of undesired motions in rough seas. Seasickness level of passengers on board should be reduced sufficiently for passenger comfort and crew operability. The first experimental studies that reveal the influence of ship vertical accelerations on the

5 human body were conducted by O'Hanlon and McCauley in 1974 1]. This experimental work was carried out with a large group of voluntary male students. Subjects were undergone different amplitude of acceleration at different oscillation frequencies in order to observe vomiting tolerance. According to the results of this work, number of graphs were plotted describing the seasickness regions by means of the frequency and amplitude of the oscillation. Additionally, it is understood that the most dangerous frequency to the human 10 body is in the vicinity of $1 \mathrm{rad} / \mathrm{s}$ and seasickness incident is proportional to the exposure time. Since most wave frequencies at sea are observed in the vicinity of $1 \mathrm{rad} / \mathrm{s}$, reducing the pitch motion of the ship by a control system is required for safer operations in a seaway.

The techniques used to reduce the pitch motion of ships have been primarily passive. However, using passive methods has significantly increased the resistance of the ship [2], 3], 4]. Hence, it is understood 15 that when the forward speed of the ship is higher than $10-15$ knots, active actuators such as fins yielded more effective solutions [5, 6].

It is well known that controller design plays a significant role in ship motion control. Therefore, a large variety of control approaches for reducing the vertical motions of ships have been presented in the literature. A proportional, integral, derivative (PID) based controller with the aim of reducing pitch and heave acceleration for a high-speed ferry form using flap and T-foil is proposed in [7. An experimental campaign was carried out in [8] to demonstrate the effectiveness of active control to damp the vertical motion of a scaled down replica of a fast ferry. In this study, vertical accelerations which are the main cause of seasickness has been reduced with a fuzzy control system based on the fuzzy model of a ship is proposed in [9]. A new model-free control approach to reduce the vertical motions induced by random waves on a high-speed ferry was also introduced at [10, 11]. In these studies, a comparison study between Proportional-Derivative (PD) and so-called i-PD (intelligent PD) was performed and the results showed that i-PD exhibited a better ability to handle the varying system parameters and operating velocity. On the other hand, Zhang et al. proposed a $\mathcal{H}_{\infty}$ output feedback control method using Ricatti equations to reduce the longitudinal motion and the sickness incidence of the wave piercing catamaran. They used instantaneous heave and pitch velocities as feedback signals [12. 6] applied a numerical and experimental study for pitch stabilization in head waves. In this work, a short term predictor has been proposed to predict hydrodynamic forces. Then these predicted motions are used in a force estimator to forecast the ship's hydrodynamics. In a similar way, [13] studied on pitch-roll stabilization problem by active fins. However, although the proposed control strategy considers magnitude bound on the angle-of-attack, it does not consider any rate 

constraint on the control signal which makes it difficult to realise on a full-sized ship having huge control fins with very large time constants. Similarly, a Linear Matrix Inequality (LMI) based robust static output feedback $\mathcal{H}_{\infty}$ controller design was developed in 14 to mitigate vertical acceleration of a motor yacht form. Among these aforementioned works, it is apparently seen that to the best of our knowledge, no study exists in literature utilizing the MPC strategy for reducing vertical ship motion by the use of anti-pitching.

40 Moreover, actuator amplitude and rate saturation problems have never been jointly taken into consideration in controller design as provided in this paper. These investigations motivate us to develop a practically applicable model predictive controller for ships having actuator amplitude and fin velocity saturation. It is noted that the optimal $\mathcal{H}_{\infty}$ controller is also applied in this paper in order to have comparative results.

In this work, a disturbance attenuation type discrete-time MPC under different wave conditions and ship forward speeds is proposed which considers actuator amplitude and rate saturation. The popularity of MPC arises from the fact that the resulting control action respects all the system and problem information, in conjunction with interactions and constraints of the system parameters, which would be very difficult to achieve by any other controller [15]. The bounds on the rate of the actuator force (torque, thrust, etc.) have also been identified as a source of severe performance degradation or instability in control applications and might yield critical limitations on the system[16. Different from the literature, the aim of this study is to develop a more realistic, practically implementable optimal controller which attenuates vertical motions of a passenger ship subject to irregular wave disturbances by respecting actuator constraints.

This paper is organized in a way to develop from modelling of a ship and wave disturbance to the MPC design for the active foil system having actuator amplitude and rate saturation. Therefore, Section 2 describes the mathematical model of the ship and the wave disturbance. Disturbance rejection type MPC strategy is developed in Section 3. Section 4 considers the design of a discrete-time state feedback $\mathcal{H}_{\infty}$ controller for systems having magnitude and rate based actuator limitations. Section 5 provides extensive simulations on the system under different sea conditions and ship speeds. Finally, Section 6 concludes the paper with some final remarks on possible research directions. The paper is further structured into subsections for better readability.

Notation. Throughout the paper, a fairly standard notation is used. The symbol $\mathbb{R}$ denotes the set of real numbers, $\mathbb{R}^{m \times n}$ stands for $m \times n$ matrices having real entries. Column vectors having $n$ entries are represented by $\mathbb{R}^{n}$. The symbol ${ }^{\wedge}$ is used to represent estimated signals. $\|z\|_{Y}^{2}$ stands for the quadratic term $z^{T} Y z .0_{m \times n}$ denotes a rectangular null matrix having a size $m \times n$ whereas $I_{n}$ represents a square $n \times n$ identity matrix. Lower case italic letters are generally used to represent vectors whereas capital italic letters are used for matrices. An ellipsoidal set having a weighting matrix $P=P^{T} \succ 0$ and centred at the origin is defined as

$$
\operatorname{Elp}(P) \triangleq\left\{x \mid x^{T} P x \leq 1\right\}
$$




\section{2. Mathematical Model of a Passenger Ship}

During the derivation of the mathematical model, Cummins' equation is used to represent the vertical ship motions of the considered passenger ship subject to irregular waves. First, the frequency domain coefficients are calculated using an in house code based on strip theory. Then, the convolution integrals used in the equation of motions are approximated by a well-known time-domain identification method. Finally, irregular head wave scenario is realized with wave-based excitation signals by the randomization theory.

\subsection{Ship Model}

In this study, we consider a passenger ship of $L_{W L}=42.95$ metres waterline length, 5.3 metres in breadth, 205 tons of mass and $2011.05 k N$ of displacement force. In the static condition, the location of the centre of gravity (LCG) is assumed to be the same as the centre of buoyancy (LCB) which is 18.67 metres from the transom. The mass inertia moment for pitch motion, $\mathcal{I}_{5}=23385$ tonm $^{2}$ and the advance velocity $V$ of the ship is assumed to be $8.2304 \mathrm{~m} / \mathrm{s}$ and $10.288 \mathrm{~m} / \mathrm{s}$. Figure 1 shows the sketch of the ship, excited with irregular long-crested head waves, in the global coordinate axis. The direction of the ship longitudinal axis is aligned to the opposite direction of the incoming waves. So the heading angle is $2 \pi$ for the head waves. Note that, in this paper, heave motion is negative from the origin downwards while pitch motion is negative when the bow of the ship goes up.

\subsection{Cummins equation for coupled heave and pitch motions}

Vertical motions of ship in a rough sea condition having a constant forward speed $V$ can be modelled by using Cummins' Equation [17] as follows:

$$
\left(\bar{M}+A^{\infty}\right) \ddot{z}(t)+B(V) \dot{z}(t)+\int_{0}^{t} K(t-\tau) \dot{z}(\tau) d \tau+C z(t)=F_{E}(t)
$$

where, $\bar{M}$ stands for the ship mass, $A^{\infty}$ denotes the mass matrix at the infinite frequency, $B(V)$ is the constant damping matrix, $C$ is the restoring matrix which is a function of only geometry, $z(t)$ is the oscillatory response of the ship and finally $F_{E}(t)$ is the transient wave force vector that can be created by a linear superposition of frequency domain results based on different wave spectra. On the other hand, $K(t)$ is the impulse response function matrix which is defined as

$$
K(t)=\frac{2}{\pi} \int_{0}^{\infty}\left[B\left(\omega_{e}\right)-B(V)\right] \cos \left(\omega_{e} t\right) d \omega_{e}
$$


Figure 1: The sketch of a ship under head wave excitation.

Here, $B\left(\omega_{e}\right)$ is the damping matrix in frequency domain. $\omega_{e}$ denotes the encounter frequency whose value can be obtained by the equation, $\omega_{e}=\omega+(k V)$ for the head waves where $\omega$ denotes the wave frequency and $k$ denotes the wave number.

Then the coupled Cummins' Equations for the vertical motion of the ship advancing with a constant speed V can be re-written as follows:

$$
\begin{aligned}
\left(\bar{M}+A_{33}^{\infty}\right) \ddot{z}_{3}(t)+B_{33}(V) \dot{z}_{3}(t) & +\int_{0}^{t} K_{33}(t-\tau) \dot{z}_{3}(\tau) d \tau+C_{33} z_{3}(t) \\
& +A_{35}^{\infty} \ddot{z}_{5}(t)+B_{35}(V) \dot{z}_{5}(t)+\int_{0}^{t} K_{35}(t-\tau) \dot{z}_{5}(\tau) d \tau+C_{35} z_{5}(t)=F_{3}(t)
\end{aligned}
$$




$$
\begin{aligned}
\left(\mathcal{I}_{5}+A_{55}^{\infty}\right) \ddot{z}_{5}(t)+B_{55}(V) \dot{z}_{5}(t) & +\int_{0}^{t} K_{55}(t-\tau) \dot{z}_{5} d(\tau)+C_{55} z_{5}(t) \\
& +A_{53}^{\infty} \ddot{z}_{3}(t)+B_{53}(V) \dot{z}_{3}(t)+\int_{0}^{t} K_{53}(t-\tau) \dot{z}_{3}(\tau) d \tau+C_{53} z_{3}(t)=F_{5}(t)
\end{aligned}
$$

${ }_{85}$ Here, $z_{3}(t), \dot{z}_{3}(t), \ddot{z}_{3}(t)$ are the heave motion, heave velocity and heave acceleration of the ship whereas $z_{5}(t), \dot{z}_{5}(t), \ddot{z}_{5}(t)$ are the pitch motion, pitch velocity and pitch acceleration of the ship, respectively. $F_{3}(t)$ represents the heave force based on irregular waves whereas $F_{5}(t)$ represents the pitch moment based on irregular waves in time domain. Further, $\mathcal{I}_{5}$ is the ship inertia moment for pitch motion. $A_{i j}^{\infty}$ is the added mass coefficient at infinite frequency. $K_{i j}(t)$ is the impulse response function whereas $C_{i j}$ is the restoring coefficient for the motion in mode $i$ with respect to the motion in the mode $j . B_{i j}(V)$ appears in Cummins' equation if the ship has a forward speed. Note that these terms have approximated values at infinite frequency and their values are obtained using the Riemann-Lesbesque Lemma [18]. Therefore, $B_{i j}(V)=B_{i j}^{\infty}$ where $i=\{3,5\}$ and $j=\{3,5\}$. The value of $A_{i j}^{\infty}$ is dependent only on the ship geometry and can be obtained by the convergence value from the frequency domain graphs. Similarly, restoring values,

${ }_{95} C_{i j}$ are also dependent on the ship geometry and can be calculated with the geometric properties of the ship.

\subsection{Calculation of Radiation Terms}

$K_{i j}(t)$ is the impulse response function which can be calculated as

$$
K_{i j}(t)=\frac{2}{\pi} \int_{0}^{\infty}\left[B_{i j}\left(\omega_{e}\right)-B_{i j}^{\infty}\right] \cos \left(\omega_{e} t\right) d \omega_{e} \quad i, j=\{3,5\}
$$

where the damping coefficient, $B_{i j}\left(\omega_{e}\right)$ is obtained by using strip theory implemented in an in-house code as shown in Figure 2, Note that the definite integrals for impulse response functions defined in (6) are calculated in the encounter frequency range and their variations are demonstrated in Figure 3 . In the present study, the definite integrals for impulse response functions defined above are calculated for a particular frequency range, $0.6-4 \mathrm{rad} / \mathrm{s}$. The frequencies lower than $0.6 \mathrm{rad} / \mathrm{s}$ and higher than $4 \mathrm{rad} / \mathrm{s}$ are ignored because the ship has almost zero response beyond this frequency range. Please note that the impulse response functions are chosen identical for the ship advance speeds which are $8.2304 \mathrm{~m} / \mathrm{s}$ and $10.288 \mathrm{~m} / \mathrm{s}$ since the encounter frequency range is set sufficiently large. Hence,

$$
K_{i j}(t) \cong \frac{2}{\pi} \int_{0.6}^{4}\left[B_{i j}\left(\omega_{e}\right)-B_{i j}^{\infty}\right] \cos \left(\omega_{e} t\right) d \omega_{e}
$$

However, it is still a very time-consuming process to calculate (4) and (5) due to the existence of the 

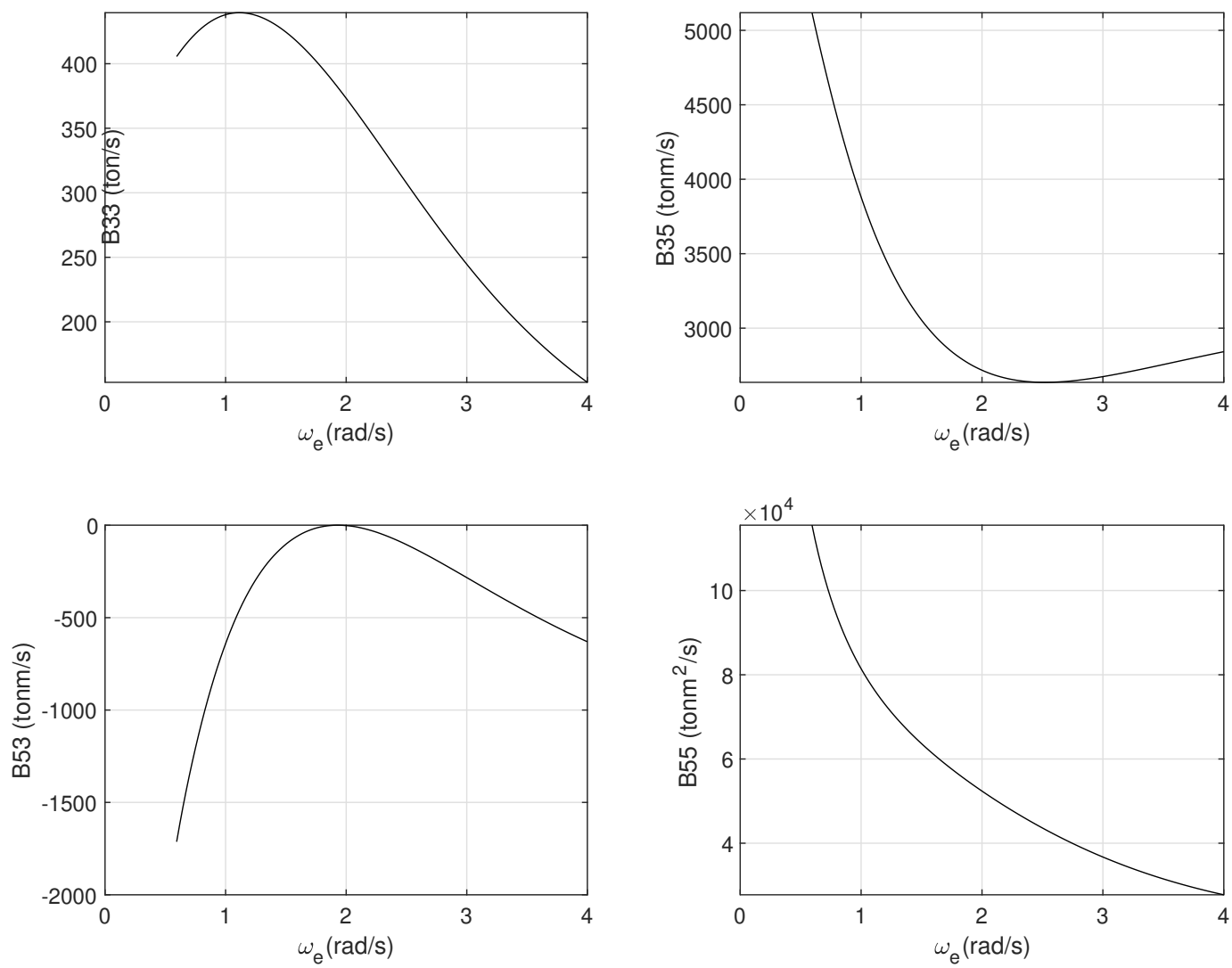

Figure 2: Damping- $w_{e}$ relations.

following terms which include convolutions:

$$
\begin{aligned}
& \eta_{1}(t) \triangleq \int_{0}^{t} K_{33}(t-\tau) \dot{z}_{3} d(\tau)+\int_{0}^{t} K_{35}(t-\tau) \dot{z}_{5} d(\tau) \\
& \eta_{2}(t) \triangleq \int_{0}^{t} K_{53}(t-\tau) \dot{z}_{3} d(\tau)+\int_{0}^{t} K_{55}(t-\tau) \dot{z}_{5} d(\tau)
\end{aligned}
$$

Note that the direct implementations of these convolution integrals, as underlined above, are computationally expensive, especially for the control applications such as model predictive control where a huge amount of information is being processed in real-time. Therefore, in this study, these convolution terms are approximated with suitable transfer functions. Following [19], in this note, filters that are obtained by using Prony's method are utilised to approximate each impulse function. Prony's method is a technique for modelling discrete data as a linear combination of exponentials. Although it cannot be classified as a spectral estimation technique, it has a close relationship with the least squares based system identification algorithms [20. Using the Prony's method along with system identification techniques, one can obtain the 

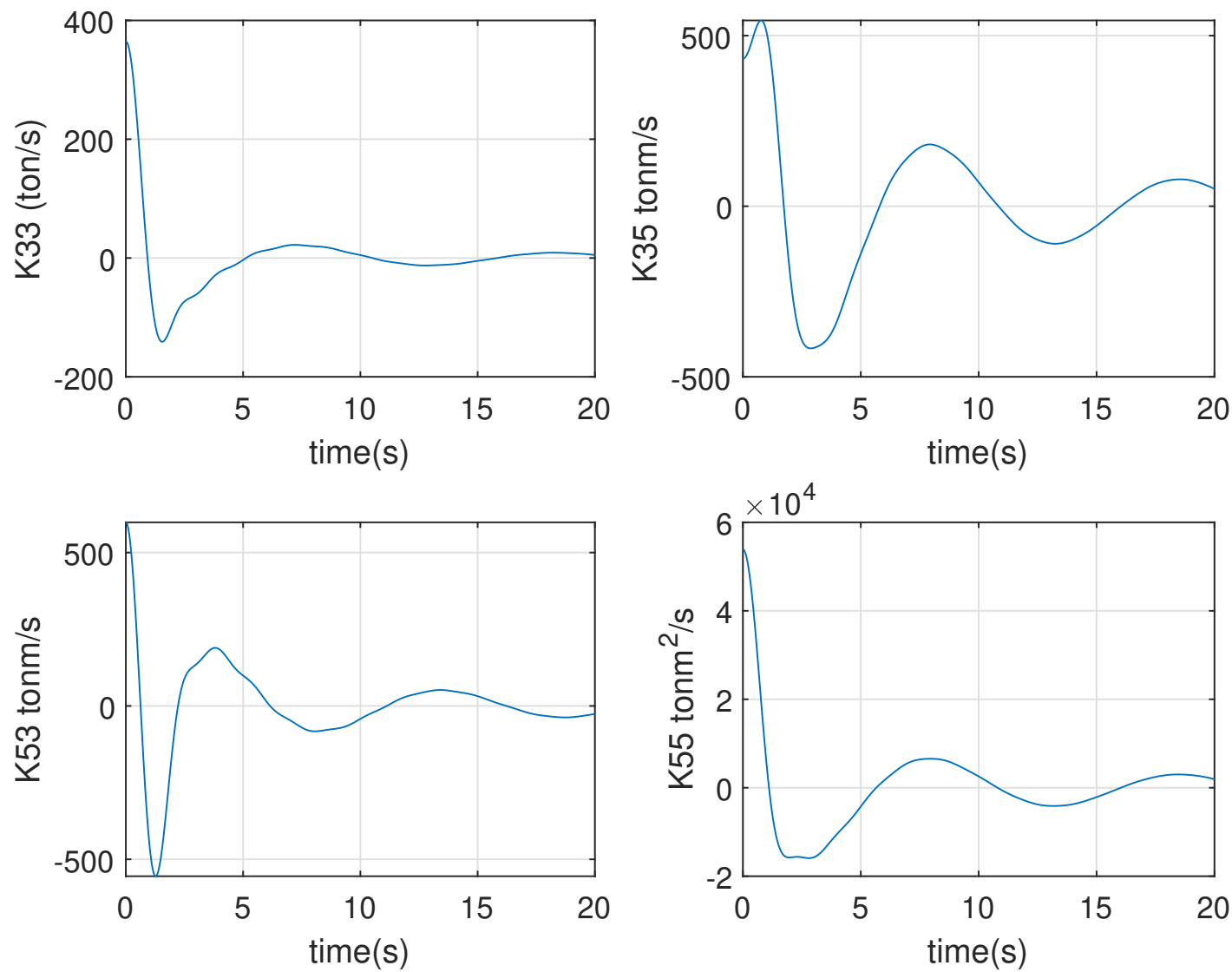

Figure 3: Impulse Response Functions.

approximated transfer functions for $K_{33}, K_{35}, K_{53}$ and $K_{55}$ as follows:

$$
\begin{gathered}
K_{33}(s) \cong \frac{364.51 s^{5}-1810.8 s^{4}+3598.9 s^{3}-3576.7 s^{2}+1777.6 s-353.42}{s^{6}-5.96 s^{5}+14.84 s^{4}-19.68 s^{3}+14.68 s^{2}-5.84 s+0.96} \\
K_{35}(s) \cong \frac{432.47 s^{5}-2156.3 s^{4}+4301.1 s^{3}-4290.4 s^{2}+2140.2 s-427.13}{s^{6}-5.98 s^{5}+14.93 s^{4}-19.86 s^{3}+14.86 s^{2}-5.93 s+0.98} \\
K_{53}(s) \cong \frac{598.49 s^{5}-2981.2 s^{4}+5940.6 s^{3}-5919.6 s^{2}+2949.6 s-587.96}{s^{6}-5.98 s^{5}+14.90 s^{4}-19.81 s^{3}+14.81 s^{2}-5.91 s+0.98} \\
K_{55}(s) \cong \frac{53875.3 s^{5}-267779.8 s^{4}+532457.01 s^{3}-529444.10 s^{2}+263259.89 s-52368.2}{s^{6}-5.97 s^{5}+14.85 s^{4}-19.71 s^{3}+14.71 s^{2}-5.85 s+0.97}
\end{gathered}
$$

As it can be seen from Figure 4 the Prony approximations represent the retardation functions with very high accuracy, particularly at the first instants of the impulse response. In this work, following a few trials, we decided to use a $6^{\text {th }}$ order approximation. However, more accurate results could be obtained by using higher order filters albeit at a high computational cost. 

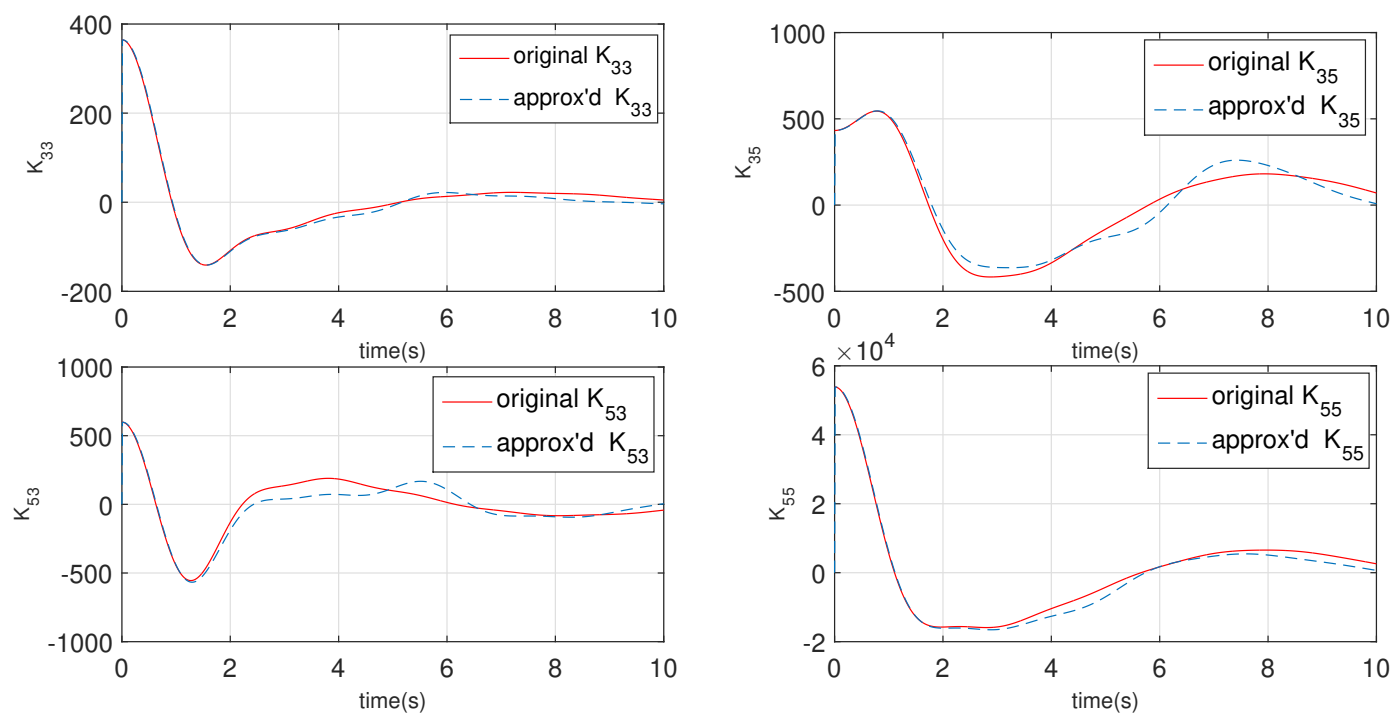

Figure 4: Representation of Impulse Response Functions with LS Method.

\subsection{Prediction of Excitation Terms}

It is very common to define a wave spectrum in order to represent the behaviour of ocean waves. If there are sufficient data from wave ride bouys, the wave spectrum can be obtained by spectral estimation techniques [5]. If these measurements are not available directly, then the idealized wave spectrum formulae can be used. In this paper, Pierson-Moskowitz spectrum, one of the most famous idealised spectrum to define the distribution of energy with frequency within the ocean, is applied.

Pierson-Moskowitz spectrum [21] can expressed as

$$
S_{\zeta}(\omega)=A \omega^{-5} e^{-\frac{B}{\omega^{4}}}
$$

where the frequency domain is used to represent excitation terms. Here, $\omega$ stands for the frequency in radians per second whereas $S_{\zeta}(\omega)$ denotes the encounter wave energy spectrum. In line with the physical parameters listed in Table 2 one can obtain the constants $A$ and $B$ as follows:

$$
\begin{gathered}
A=0.0081 \times g^{2} \\
B=\frac{0.032 g^{2}}{H_{s}^{2}}
\end{gathered}
$$

where $H_{s}$ denotes the characteristic wave height and $g$ denotes the acceleration of gravity. In this study, three different significant wave height conditions (0.70, 0.88 and 1.0 metres) were considered during the simulation of irregular waves. For the excitation term predictions, the linear superposition principle which was proposed in 22] is used. Hence,

$$
S_{\mathrm{HF} / \mathrm{PM}}\left(\omega_{e}\right)=S_{\zeta}\left(\omega_{e}\right) \times\left|T F_{\mathrm{HF} / \mathrm{PM}}\left(\omega_{e}\right)\right|^{2}
$$




$$
S_{\zeta}\left(\omega_{e}\right)=\frac{S_{\zeta}(\omega)}{1+\frac{2 V \omega}{g}}
$$

For example, wave encounter energy spectrum $S_{\zeta}\left(\omega_{e}\right)$, is plotted in Figure 5 for $H_{s}=0.88$. In 16 , $T F_{\mathrm{HF} / \mathrm{PM}}\left(\omega_{e}\right)$ denotes the heave force or pitch moment in regular head waves while the ship has a forward speed $V=10.288 \mathrm{~m} / \mathrm{s}$ and they are given in Figure 6. Please note that different ship advance speeds refer to different wave excitations under the same wave conditions. On the other hand, $S_{\mathrm{HF} / \mathrm{PM}}\left(\omega_{e}\right)$ stands for the heave force or pitch moment response spectrum for the investigated wave. According to linear random

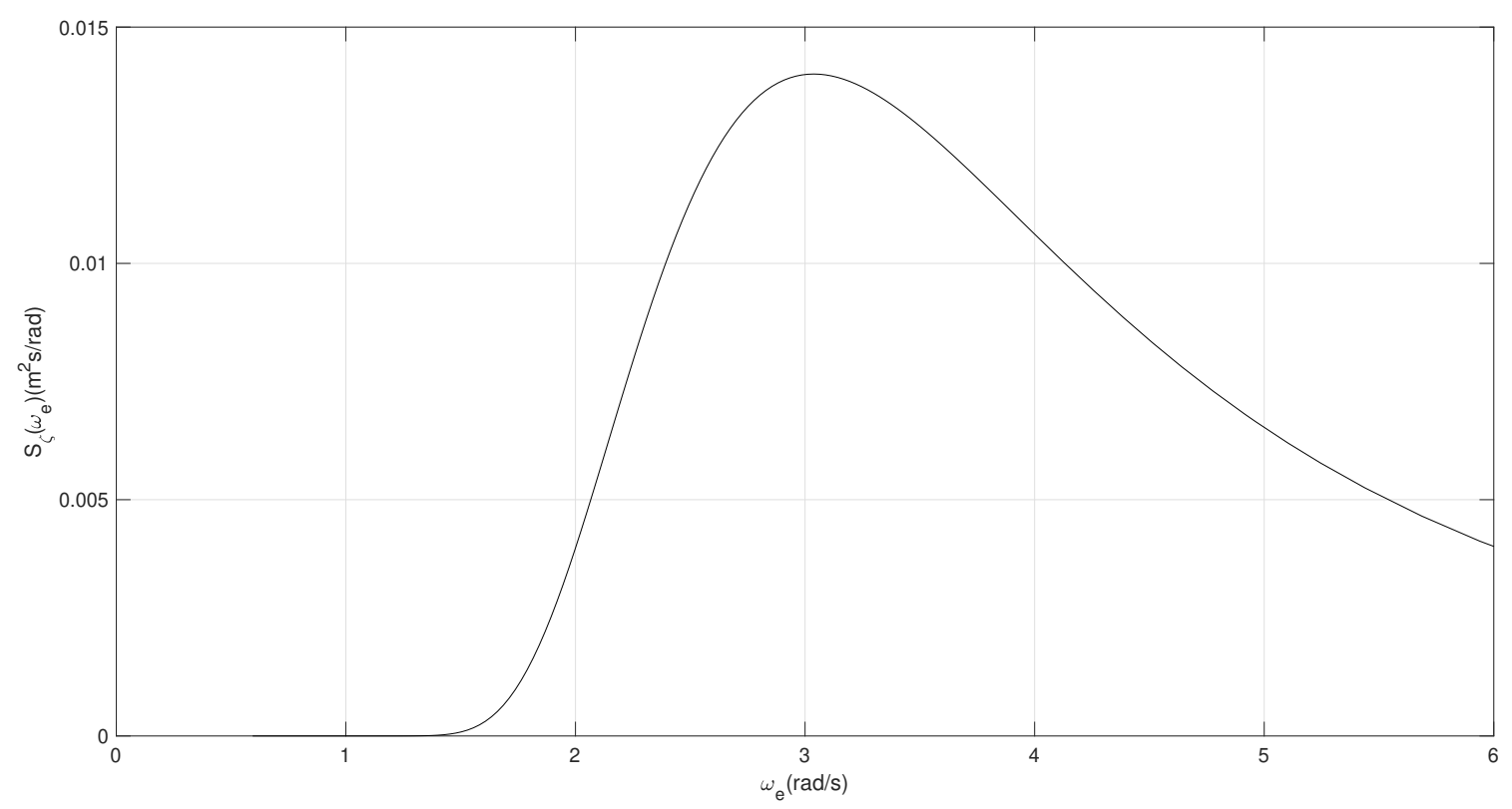

Figure 5: Wave encounter energy spectrum $S_{\zeta}\left(\omega_{e}\right)$ for $H_{s}=0.88 \mathrm{~m}$.

wave theory, unidirectional and long-crested waves can be expressed by the sum of finite regular waves. Therefore, instantaneous wave amplitude can be stated as

$$
\zeta(x, t)=\sum_{i=1}^{N_{i}} A_{i} \sin \left(\theta_{i}(t)\right)
$$

where $\zeta(x, t)$ denotes the mean wave amplitude measured from the free surface and $A_{i}=\sqrt{2 S_{\zeta}\left(\omega_{i}\right) \Delta \omega_{i}}$ denotes wave amplitude of each frequency component. In this expression, $\theta_{i}(t)=k_{i} x+\omega_{i} t+\varepsilon_{i}$, where $t$ denotes the time, $\omega_{i}$ denotes the $i^{\text {th }}$ frequency and $\varepsilon_{i}$ denotes the $i^{\text {th }}$ phase lag whose value is randomly assigned between 0 and $2 \pi$. Also $\Delta \omega_{i}$ is the $i^{\text {th }}$ step size of the frequency. $x$ denotes the position of the wave and $k_{i}$ denotes $i^{\text {th }}$ wave number. By taking the wave position (i.e $x_{i}=0$ for all $i$ ) and following the same procedure, the heave force or pitch moment response spectra, shown in Figure 7 can be transformed to the 

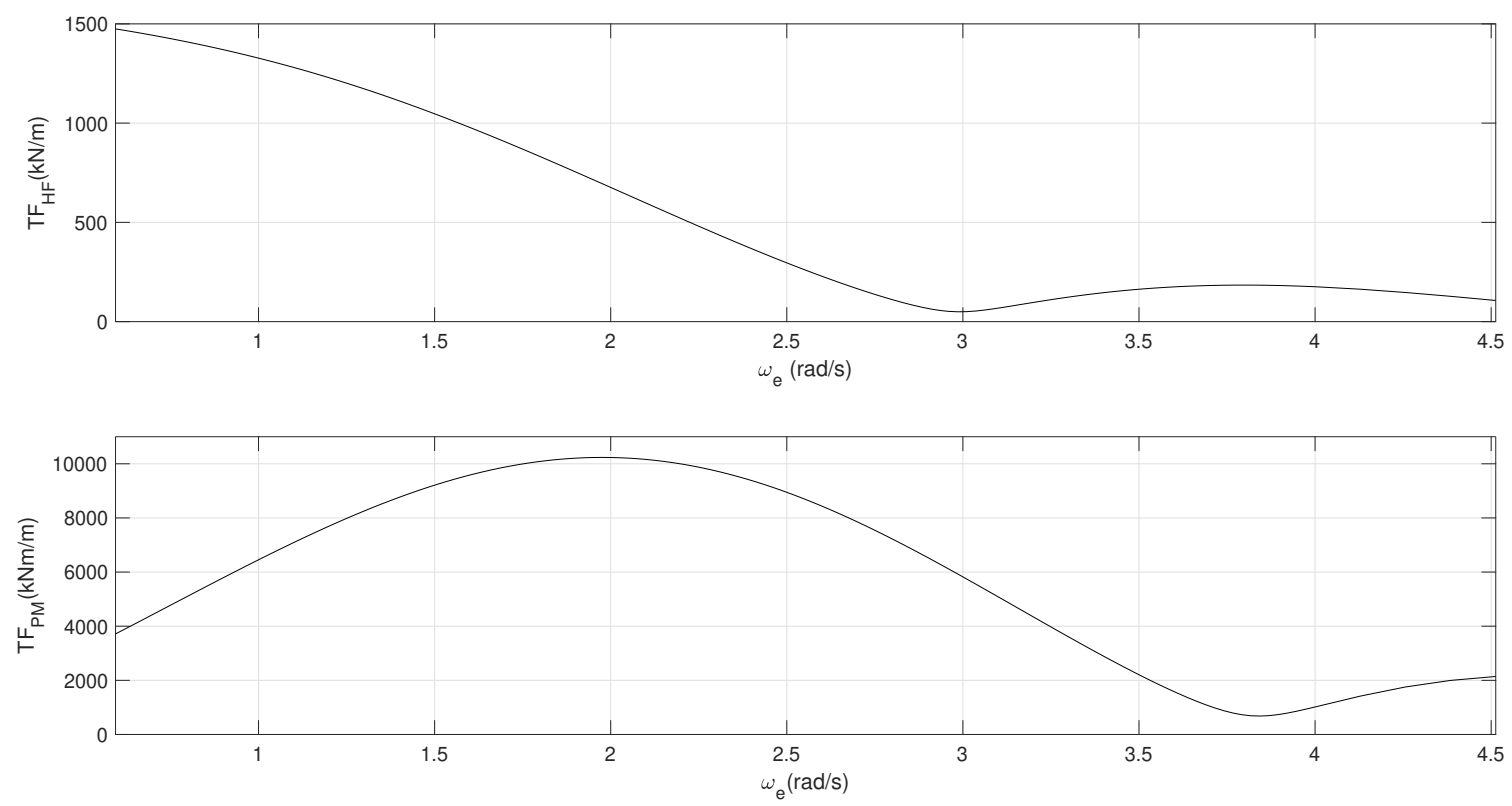

Figure 6: Heave force and pitch moment $T F$ at $V=10.288 \mathrm{~m} / \mathrm{s}$.
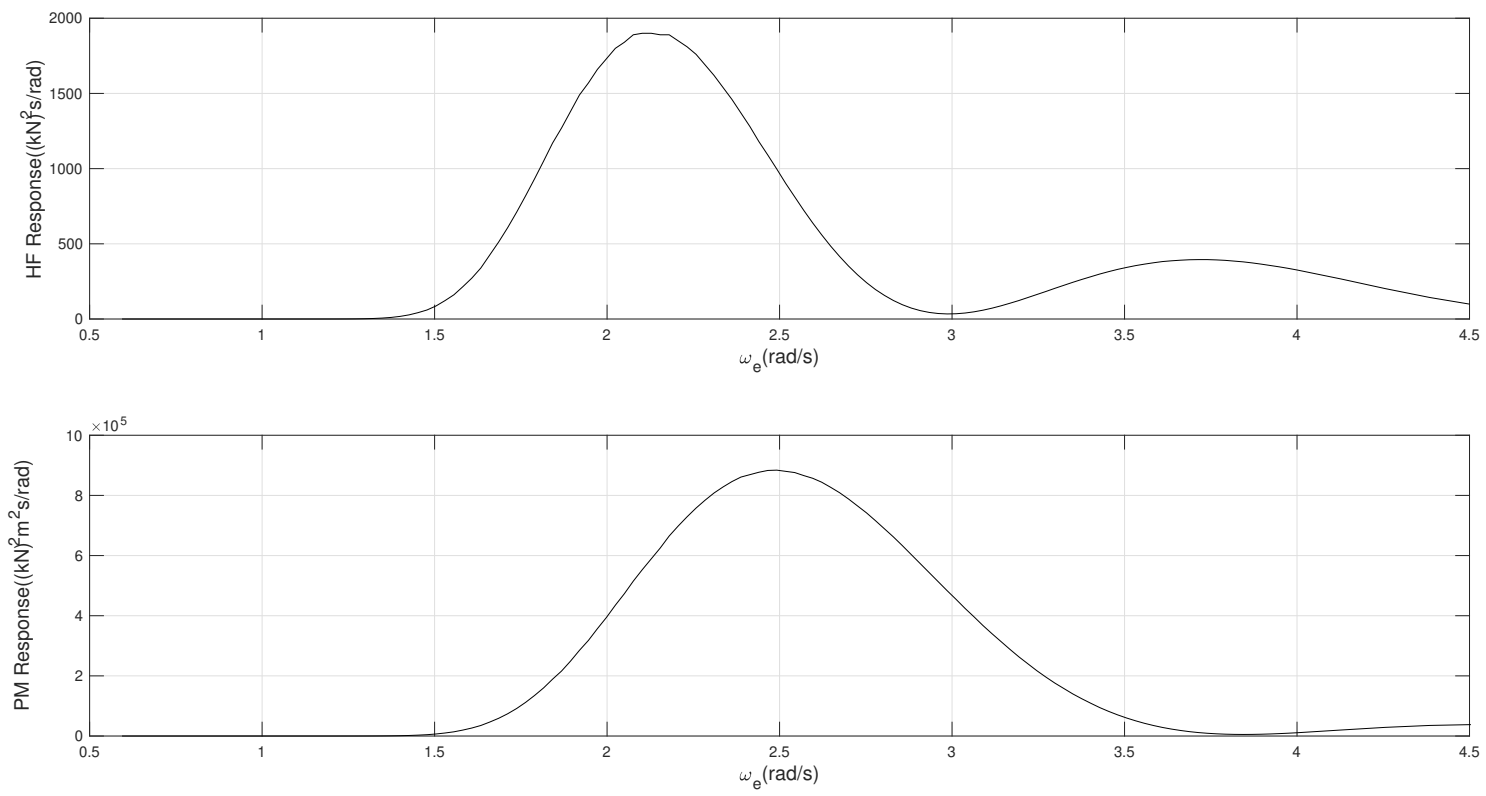

Figure 7: Heave Force-Pitch Moment Response Spectra representing $H_{s}=0.88 \mathrm{~m}$. at $V=10.288 \mathrm{~m} / \mathrm{s}$.

time domain as shown in Figure 8 for a simulation that lasts 100 seconds [5]. In this work, the number of 
sufficiently enough to represent heave force and pitch moment due to wave-ship interactions.
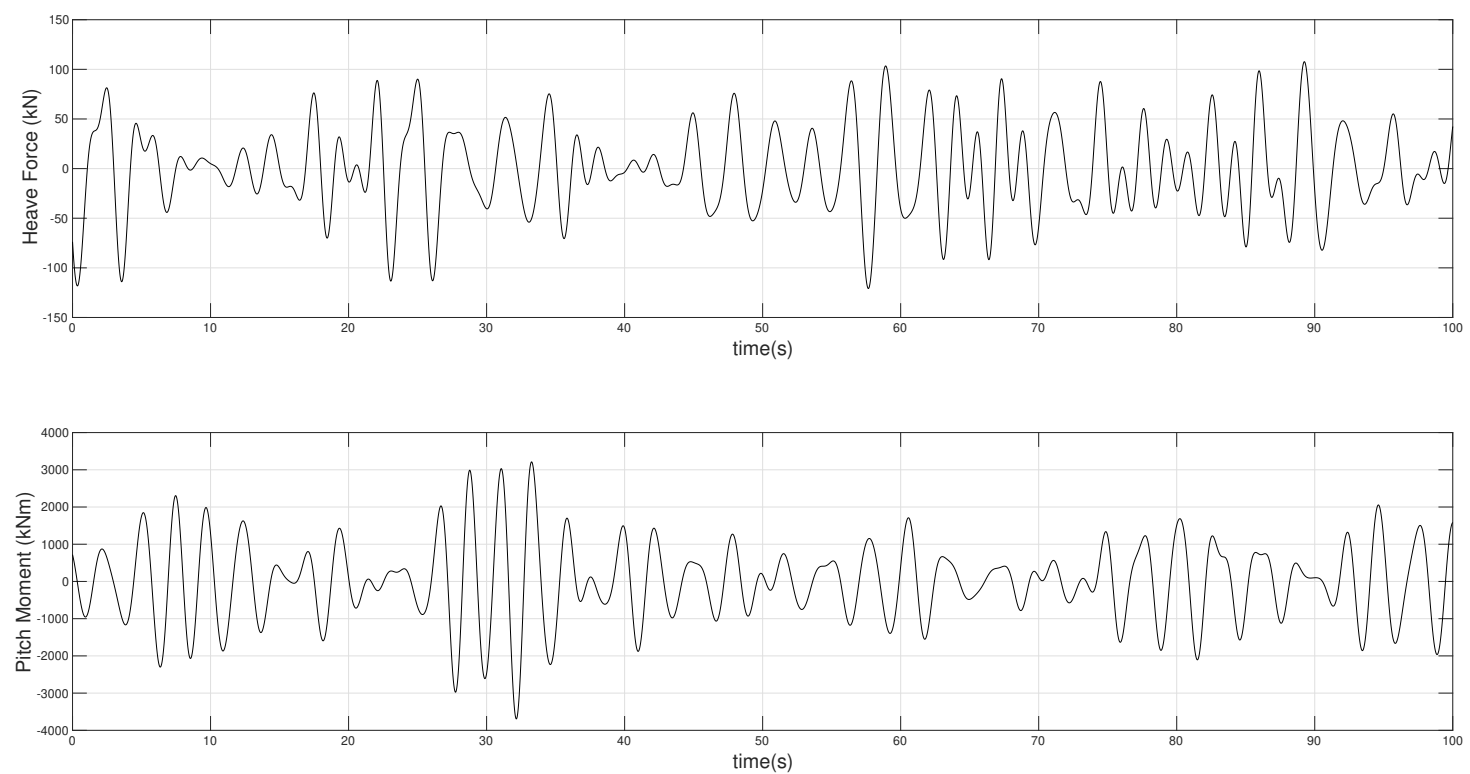

Figure 8: Heave Force and Pitch Moment in Time Domain at Hs=0.88 m.at $V=10.288 \mathrm{~m} / \mathrm{s}$.

\subsection{State Space Representation of Mathematical Model of Vertical Ship Motions and Actuator Equipped with Anti-Pitching foils}

In this study, state-space representation is used to define the model of the system due to its multi-input and multi-output structure. The vertical motion of the ship can be represented as

$$
\dot{x}(t)=A x(t)+B_{1}[\operatorname{wave}(t)-\operatorname{conv}(t)]+B_{2} \alpha(t)
$$

where $x(t) \in \mathbb{R}^{n}$ denotes the differentiable state vector, wave $(t) \in \mathbb{R}^{m_{w}}$ denotes the wave load input vector, $\operatorname{conv}(t) \in \mathbb{R}^{m_{w}}$ denotes the convolution vector, and $\alpha(t) \in \mathbb{R}^{m_{u}}$ denotes the control input vector. $A, B_{1}$ and $B_{2}$ are known state-space matrices with appropriate dimensions. Using the change of variables, $x_{1}(t) \triangleq z_{3}(t)$, $x_{2}(t) \triangleq z_{5}(t), x_{3}(t) \triangleq \dot{z}_{3}(t)$ and $x_{4}(t) \triangleq \dot{z}_{5}(t)$, the system matrices, disturbance input, control input and 
states of the system can be obtained as follows:

$$
\begin{aligned}
& A=\left[\begin{array}{cc}
0_{2 \times 2} & I_{2} \\
-M^{-1} R & -M^{-1} D
\end{array}\right], B_{1}=\left[\begin{array}{c}
0_{2 \times 2} \\
M^{-1}
\end{array}\right] \\
& B_{2}=\left[0.5 \times \rho \times V^{2} \times(180 / \pi) \times\left[\begin{array}{cc}
-1 & -1 \\
m_{b} & -m_{s}
\end{array}\right]\left[\begin{array}{cc}
C_{\text {Lbow }} & 0 \\
0 & C_{\text {Lstern }}
\end{array}\right]\left[\begin{array}{cc}
S_{\text {bow }} & 0 \\
0 & S_{\text {stern }}
\end{array}\right] \times M^{-1}\right],
\end{aligned}
$$

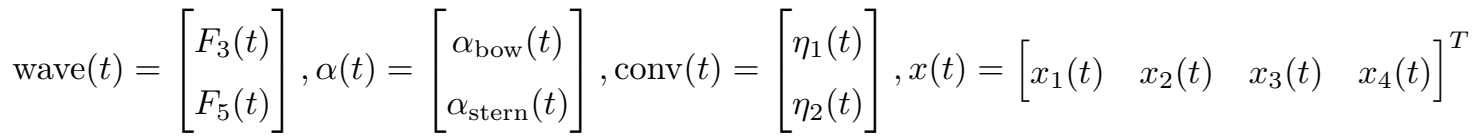

where, $x_{1}(t)$ denotes the heave motion, $x_{2}(t)$ stands for the pitch motion, $x_{3}(t)$ represents the heave velocity and $x_{4}(t)$ symbolises the pitch velocity. $F_{3}(t)$ denotes the heave force due to waves, $F_{5}(t)$ is used to represent the pitch moment due to waves. The terms aforementioned in the state-space matrices are as follows:

$$
M=\left[\begin{array}{cc}
\bar{M}+A_{33}^{\infty} & A_{53}^{\infty} \\
A_{53}^{\infty} & \mathcal{I}_{5}+A_{55}^{\infty}
\end{array}\right], \quad R=\left[\begin{array}{cc}
C_{33} & C_{35} \\
C_{53} & C_{55}
\end{array}\right], \quad D=\left[\begin{array}{cc}
B_{33}^{\infty} & B_{35}^{\infty} \\
B_{53}^{\infty} & B_{55}^{\infty}
\end{array}\right]
$$

\begin{tabular}{|c|c|}
\hline Parameter (Unit) & Value \\
\hline$M \quad($ ton $)$ & 205 \\
\hline $\mathcal{I}_{5}\left(\right.$ tonm $\left.^{2}\right)$ & 23385 \\
\hline$A_{33}^{\infty} \quad($ ton $)$ & 214 \\
\hline$A_{35}^{\infty}($ tonm $)$ & 560 \\
\hline$A_{53}^{\infty}($ tonm $)$ & 568 \\
\hline$A_{55}^{\infty}\left(\right.$ tonm $\left.^{2}\right)$ & 19752 \\
\hline$B_{33}^{\infty} \quad($ ton $/ \mathrm{s})$ & 153 \\
\hline$B_{35}^{\infty}($ tonm $/ \mathrm{s})$ & 2841 \\
\hline$B_{53}^{\infty}($ tonm $/ \mathrm{s})$ & -630 \\
\hline$B_{55}^{\infty}\left(\right.$ tonm $\left.^{2} / s\right)$ & 27752 \\
\hline$C_{33}\left(\operatorname{ton} / \mathrm{s}^{2}\right)$ & 1566 \\
\hline$C_{35}\left(\right.$ tonm $\left./ \mathrm{s}^{2}\right)$ & 1287 \\
\hline$C_{53}\left(\right.$ tonm $\left./ \mathrm{s}^{2}\right)$ & 1287 \\
\hline$C_{55}\left(\right.$ tonm $\left.^{2} / \mathrm{s}^{2}\right)$ & 173322 \\
\hline
\end{tabular}

It should be noted that in $B_{2}$ matrix, moment arms $m_{b}$ and $m_{s}$ were taken as 19.25 metres from the bow

and 16.80 metres from the stern with respect to centre of gravity. In addition to this, $M$ denotes the mass matrix, $R$ denotes the restoring matrix and $D$ denotes the damping matrix. The passenger ship is equipped 
with two submerged anti-pitching active foils. Table 1 summarizes the model parameters and their physical values used in this note. As shown in Figure 1, a pair of foils having symmetrical sections situated at bow and stern part of the ship to reduce the vertical motions. These foils are used to control vertical motions of the ship body in accordance to the control forces $U_{\text {bow }}(t)$ and $U_{\text {stern }}(t)$ computed by the real-time controller. Note that these actuators produce lift force as a function of ship speed $V$, effective angle of attacks, $\alpha_{\text {bow }}(t)$ and $\alpha_{\text {stern }}(t)$, projected area of the foils $S_{\text {bow }}$ and $S_{\text {stern }}$, lift coefficients of the foils $C_{\text {Lbow }}$ and $C_{\text {Lstern }}$ and finally water density $\rho$. Note that in this work, $S_{\text {bow }}=S_{\text {stern }}=S$ and $C_{\text {Lbow }}=C_{\text {Lstern }}=C_{L}$. Then one can define

$$
\begin{gathered}
U_{\text {bow }}(t) \triangleq 0.5 \times \rho \times S_{\text {bow }} \times C_{\text {Lbow }} \times(180 / p i) \times V^{2} \times \alpha_{\text {bow }}(t) \\
U_{\text {stern }}(t) \triangleq 0.5 \times \rho \times S_{\text {stern }} \times C_{\text {Lstern }} \times(180 / p i) \times V^{2} \times \alpha_{\text {stern }}(t)
\end{gathered}
$$

Hence, 22 and 23 can be used to transform the vertical forces $U_{\text {bow }}(t)$ and $U_{\text {stern }}(t)$ into effective foil angles, $\alpha_{\text {bow }}(t)$ and $\alpha_{\text {stern }}(t)$ assuming that the impact of the unsteady behaviour of lift characteristics is negligible. Here, the angle of attacks, $\alpha_{\text {bow }}(t)$ and $\alpha_{\text {stern }}(t)$ should be measured with respect to the parallel water flow. Therefore, the real angle of attacks can be expressed in terms of the online foil angle and the position of the ship as follows:

$$
\begin{array}{r}
\alpha_{\text {bow }}(t)=\beta_{\text {bow }}(t)+x_{2}(t)+\theta_{\text {Fbow }}(t) \\
\alpha_{\text {stern }}(t)=\beta_{\text {stern }}(t)+x_{2}(t)+\theta_{\text {Fstern }}(t)
\end{array}
$$

where, $\beta_{\text {bow }}(t)$ is the bow foil angle and $\beta_{\text {stern }}(t)$ is the stern foil angle of the ship. $\theta_{\text {Fbow }}(t)$ and $\theta_{\text {Fbow }}(t)$ depict the change the angle of attacks due to the online pitch and heave velocities of the ship and can be expressed as follows assuming that the arguments of the arctan functions vary in a very small interval:

$$
\begin{gathered}
\theta_{\text {Fbow }}(t)=\arctan \left(\frac{m_{b} \times x_{4}-x_{3}+\dot{\xi}}{V}\right) \cong\left(\frac{m_{b} \times x_{4}-x_{3}+\dot{\xi}}{V}\right) \\
\theta_{\text {Fstern }}(t)=\arctan \left(\frac{-m_{s} \times x_{4}-x_{3}+\dot{\xi}}{V}\right) \cong\left(\frac{-m_{s} \times x_{4}-x_{3}+\dot{\xi}}{V}\right)
\end{gathered}
$$

Here, $\dot{\xi}$ stands for the heave velocity of wave on hydrofoil surface and its value is neglected since the foils are positioned sufficiently deep under mean water surface. Please note that there is also a contribution coming from the drag component due to the flow passing through the foil. However, this contribution may also be ignored since it is very small compared to the lift component.

Taking the foil opening angle $u(t) \triangleq \beta(t)$ as the the actual control input, the state-space equations of the vertical motion can be expressed as

$$
\begin{aligned}
& \dot{x}(t)=\left(A+B_{2} B_{3}\right) x(t)+B_{1}[\operatorname{wave}(t)-\operatorname{conv}(t)]+B_{2} u(t) \\
& y(t)=C x(t)
\end{aligned}
$$


where

$$
C=\left[\begin{array}{llll}
0 & 1 & 0 & 0 \\
0 & 0 & 0 & 1
\end{array}\right], \quad B_{3}=\left[\begin{array}{cccc}
0 & 1 & -\frac{1}{V} & \frac{m_{b}}{V} \\
0 & 1 & -\frac{1}{V} & -\frac{m_{s}}{V}
\end{array}\right]
$$

All parameters that belong to the actuators are given in Table 2 In this paper, two identical active foils are used to actuate the system and their rotation angles are limited to $\pm 20 \mathrm{deg}( \pm 0.349 \mathrm{rad})$, and rotation rates are limited to $\pm 20 \mathrm{deg} / \mathrm{s}( \pm 0.349 \mathrm{rad} / \mathrm{s})$, due to mechanical restrictions of the actuators that drive foils [23]. On the other hand, it is well-know that the projected foil area is related to the water plane area of the ship and assessed according to a survey study for the cruise ships [23. In this study, NACA 0021 hydrofoil is selected as the foil's section. Based on 24], Computational Fluid Dynamics (CFD) calculations gives 0.047 for the lift coefficient of NACA 0021 profile, having the aspect ratio $A R=2$. This is obtained by fitting a linear curve to numerically calculated data.

Table 2: Parameters of the actuators

\begin{tabular}{lcc}
\multicolumn{3}{c}{ Table 2: Parameters of the actuators } \\
\hline \hline Parameters & Bow Foil & Stern Foil \\
\hline Span $(\mathrm{m})$ & 1.866 & 1.866 \\
Chord $(\mathrm{m})$ & 0.933 & 0.933 \\
$\mathrm{~S}\left(\mathrm{~m}^{2}\right)$ & 1.742 & 1.742 \\
AR $($ Span/Chord $)(-)$ & 2 & 2 \\
Max. angle limit $\left(u_{\min }, u_{\max }\right)(\mathrm{rad})$. & \pm 0.349 & \pm 0.349 \\
Max. rotation rate limit $(\mathrm{rad} / \mathrm{s})\left(u_{\min }^{d}, u_{\max }^{d}\right)$ & \pm 0.349 & \pm 0.349 \\
$C_{L}(-)$ & 0.047 & 0.047 \\
Distance to CoG $\left(m_{b}\right.$ and $\left.m_{s}\right)(\mathrm{m})$ & 19.25 & 16.80 \\
\hline
\end{tabular}

\section{Disturbance Rejection Based Model Predictive Controller (MPC) Design}

It is well known that input saturation, which can restrict the capability of actuators might cause remarkable performance degradation on the control system performance, sometimes even destabilization [25]. In many electro-mechanical applications including the active foil systems considered in this study, actuator can be saturated both in terms of the magnitude and the rate of change of the signal that is applied to the system. Therefore, the controllers that were designed by not considering these physical limitations cannot demonstrate the high performance that they exhibit in the simulation environment on the real system. Hence, in order to have a realistic and practically implementable controller, the design must consider the physical restrictions on both angle and the rate of the angle of foil. To overcome this issue, in this study, we propose an MPC strategy for the control of active foil system which is capable of eliminating the slowly varying disturbances such as waves by its integral type structure. 
MPC is an advanced control strategy that is used to control complex processes while satisfying a set of constraints arising from the engaged system's characteristics. The main advantage of MPC is the fact that it allows the current timeslot to be optimally controlled, while keeping future behaviour of the system timeslot and then optimizing again, repeatedly, thus differing from any other offline optimal control method. The rest of this section investigates the design of an integral type multi-objective MPC that can be used in active foil control systems in ships.

Consider a linear discrete-time state-space model of the vertical motions of a ship governed by the following difference equations:

$$
\begin{aligned}
x_{k+1} & =\mathcal{A} x_{k}+\mathcal{B} u_{k}+\mathcal{B}_{w} w_{k} \\
y_{k} & =\mathcal{C} x_{k}+v_{k}
\end{aligned}
$$

where the subscripts refer to the sampling instants, $x_{k} \in \mathbb{R}^{n}$ is the state vector, $u_{k} \in \mathbb{R}^{m}$ is the control vector, $w_{k} \in \mathbb{R}^{s}$, is the disturbance signal having effect on the states, $v_{k} \in \mathbb{R}^{p}$ is the output disturbance and $y_{k} \in \mathbb{R}^{p}$ stands for the outputs. $\mathcal{A}, \mathcal{B}, \mathcal{B}_{w}$ and $\mathcal{C}$ are the system matrices obtained by discretising the continuous-time system having system matrices $A+B_{2} B_{3}, B_{2}, B_{1}$ and $C$, using a Zero-Order-Hold (ZOH) on the inputs and a sample time of $T_{s}$ seconds, respectively. On the other hand, since the period of sea waves that affect the ship is much larger than the sampling time $T_{s}$, one can assume that the rate of change of the disturbance signals $w_{k}$ and $v_{k}$ are negligible, i.e, $w_{k} \simeq w_{k-1}$ and $v_{k} \simeq v_{k-1}$ for all $k \geq 0$. Hence, one can assume that $w_{k}=w$ and $v_{k}=v$ for all $k \geq 0$. Note that for discrete-time models used in control, there is normally no direct feed-through term $\mathcal{D} u_{k}$. Hence, the measurement $y_{k}$ does not depend on the input at time $k$, but it depends on the input at time $k-1$ through the state $x_{k}$.

The control task of this note is to design an MPC strategy so that the output $y_{k}$ follows a pre-defined reference input $r_{k}$ as close as possible for all iterations $k$ without violating the following component-wise control signal constraints:

$$
\begin{gathered}
u_{\text {min }} \leq u_{k} \leq u_{\max }, \quad \forall 0 \leq k<N-1 \\
u_{\min }^{d} \leq \underbrace{u_{k}-u_{k-1}}_{\Delta u_{k}} \leq u_{\max }^{d}, \quad \forall 0 \leq k<N-1
\end{gathered}
$$

$u_{\text {min }}$ stands for the lower bound of the control signal and $u_{\max }$ represents the upper bound of the control signal. $u_{\max }^{d}$ corresponds to the upper bound of the rate of the control signal whereas $u_{\text {min }}^{d}$ denotes the lower bound for the rate of the control signal. For our case, the numerical values of these limits are shown in Table 2, Here, $N$ is called the prediction horizon. 
Hence, the optimisation problem can be stated as follows:

$$
\underset{\Delta u_{t}, \ldots, \Delta u_{t+N-1}}{\operatorname{minimise}} J=\frac{1}{2}\left\|e_{t+N}\right\|_{S}^{2}+\frac{1}{2} \sum_{k=0}^{N-1}\left(\left\|e_{t+k}\right\|_{Q}^{2}+\left\|\Delta u_{t+k}\right\|_{R}^{2}\right)
$$

where $t$ represents the current time, $J$ is the running cost function, $e_{k} \triangleq y_{k}-r_{k}$ stands for the tracking error; $S, Q$ and $R$ denote the symmetric positive definite constant weighting matrices with appropriate dimensions.

Our goal is to find out a suitable optimal MPC controller signal array $u^{\star} \in \mathbb{R}^{N \cdot m}$ that solves the optimisation problem (33) along with system trajectory 30 . Also the solution must satisfy the input constraints (31), (32) and capable of coping with the effects of slowly varying disturbances $w$ and $v$ on the states and the outputs, respectively. To achieve this goal, let us define a new augmented state vector $\tilde{x}_{k} \triangleq\left[\begin{array}{ll}\Delta x_{k}^{T} & y_{k-1}^{T}\end{array}\right]^{T}$ where $\Delta x_{k} \triangleq x_{k}-x_{k-1}$. Hence,

$$
\begin{aligned}
\Delta x_{k+1} & =x_{k+1}-x_{k} \\
& =\mathcal{A} x_{k}+\mathcal{B} u_{k}+\mathcal{B} w w-\mathcal{A} x_{k-1}-\mathcal{B} u_{k-1}-\mathcal{B}_{w} w \\
& =\mathcal{A}\left(x_{k}-x_{k-1}\right)+\mathcal{B} \underbrace{\left(u_{k}-u_{k-1}\right)}_{\Delta u_{k}} \\
& =\mathcal{A} \Delta x_{k}+\mathcal{B} \Delta u_{k}
\end{aligned}
$$

Similarly,

$$
\begin{aligned}
y_{k}-y_{k-1} & =\mathcal{C} x_{k}+v-\mathcal{C} x_{k-1}-v \\
y_{k} & =\mathcal{C} \Delta x_{k}+y_{k-1}
\end{aligned}
$$

Then, for the sampling instant $k$, one can define the following augmented state and output equations which are reliant on the rate of change of the control signal $\Delta u_{k}$.

$$
\begin{aligned}
{\left[\begin{array}{c}
\Delta x_{k+1} \\
y_{k}
\end{array}\right] } & =\underbrace{\left[\begin{array}{cc}
\mathcal{A} & 0_{n \times p} \\
\mathcal{C} & I_{p}
\end{array}\right]}_{\tilde{A}} \underbrace{\left[\begin{array}{c}
\Delta x_{k} \\
y_{k-1}
\end{array}\right]}_{\tilde{x}_{k}}+\underbrace{\left[\begin{array}{c}
\mathcal{B} \\
0_{p \times m}
\end{array}\right]}_{\tilde{B}} \Delta u_{k} \\
y_{k} & =\underbrace{\left[\begin{array}{ll}
\mathcal{C} & I_{p}
\end{array}\right]}_{\tilde{C}} \underbrace{\left[\begin{array}{c}
\Delta x_{k} \\
y_{k-1}
\end{array}\right]}_{\tilde{x}_{k}}
\end{aligned}
$$

It is also obvious from these equations that the new augmented state-space system is independent of the disturbances, $w$ and $v$. Then, using the definitions

$$
r \triangleq\left[\begin{array}{c}
r_{t+1} \\
\vdots \\
r_{t+N}
\end{array}\right] \in \mathbb{R}^{N \cdot p}, \tilde{x} \triangleq\left[\begin{array}{c}
\tilde{x}_{t+1} \\
\vdots \\
\tilde{x}_{t+N}
\end{array}\right] \in \mathbb{R}^{N \cdot(n+p)}, \Delta u \triangleq\left[\begin{array}{c}
\Delta u_{t} \\
\vdots \\
\Delta u_{t+N-1}
\end{array}\right] \in \mathbb{R}^{N \cdot m}
$$


one can write the prediction equation as follows:

$$
\tilde{x}=\bar{C} \Delta u+\bar{A} \tilde{x}_{t}
$$

where

$$
\bar{C}=\left[\begin{array}{cccc}
\tilde{B} & 0 & \cdots & 0 \\
\tilde{A} \tilde{B} & \tilde{B} & \cdots & 0 \\
\vdots & \vdots & \ddots & \vdots \\
\tilde{A}^{N-1} \tilde{B} & \tilde{A}^{N-2} \tilde{B} & \cdots & \tilde{B}
\end{array}\right], \quad \bar{A}=\left[\begin{array}{c}
\tilde{A} \\
\tilde{A}^{2} \\
\vdots \\
\tilde{A}^{N}
\end{array}\right]
$$

Hence, in the light of these definitions, the optimisation problem 33 can be rewritten as

$$
\underset{\Delta u}{\operatorname{minimise}} J=\frac{1}{2} \Delta u^{T} H \Delta u+\left[\begin{array}{ll}
\tilde{x}_{t}^{T} & r^{T}
\end{array}\right] F \Delta u
$$

where

$$
\begin{aligned}
& H=\bar{C}^{T} \bar{Q} \bar{C}+\bar{R} \\
& F=\left[\begin{array}{c}
\bar{A}^{T} \bar{Q} \bar{C} \\
-T \bar{C}
\end{array}\right] \\
& \bar{Q}=\left[\begin{array}{cccc}
\tilde{C}^{T} Q \tilde{C} & & & \\
& \ddots & & \\
& & \tilde{C}^{T} Q \tilde{C} & \\
& & & \tilde{C}^{T} S \tilde{C}
\end{array}\right] N-1 \text { times }
\end{aligned}
$$

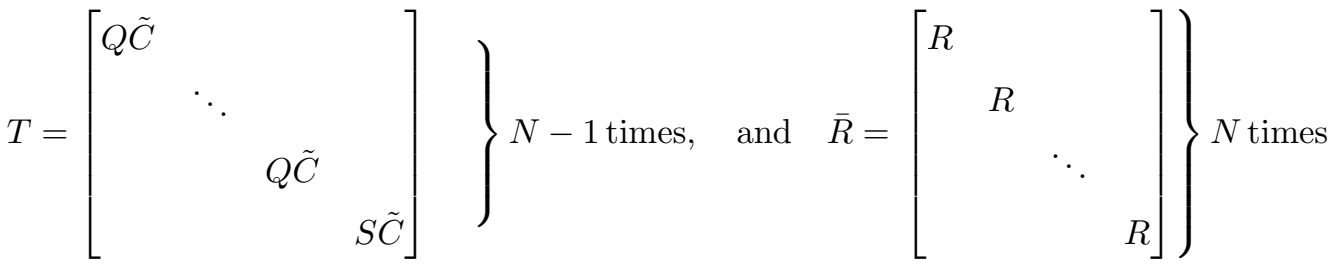

Here, $H$ is called the Hessian matrix which needs to be always positive definite. In order to utilise 40 in the MPC design, the control constraints (31) and (32) must also be defined in terms of the decision variable $\Delta u$.

Note that

$$
\left[\begin{array}{c}
u_{t} \\
u_{t+1} \\
\vdots \\
u_{t+N-1}
\end{array}\right]=\underbrace{\left[\begin{array}{c}
I_{m} \\
I_{m} \\
\vdots \\
I_{m}
\end{array}\right]}_{\tilde{I}_{m}} u_{t-1}+\underbrace{\left[\begin{array}{cccc}
I_{m} & 0 & \cdots & 0 \\
I_{m} & I_{m} & \cdots & 0 \\
\vdots & \vdots & \ddots & \vdots \\
I_{m} & I_{m} & \cdots & I_{m}
\end{array}\right]}_{\Omega_{1}} \underbrace{\left[\begin{array}{c}
\Delta u_{t} \\
\Delta u_{t+1} \\
\vdots \\
\Delta u_{t+N-1}
\end{array}\right]}_{\Delta u}
$$


Hence, 31 can be replaced with

$$
\left[\begin{array}{c}
\Omega_{1} \\
-\Omega_{1}
\end{array}\right] \Delta u \leq\left[\begin{array}{c}
U_{\mathrm{MAX}} \\
-U_{\mathrm{MIN}}
\end{array}\right]-\left[\begin{array}{c}
\tilde{I}_{m} \\
-\tilde{I}_{m}
\end{array}\right] u_{t-1}
$$

where

$$
U_{\mathrm{MAX}}=\left[\begin{array}{c}
u_{\max } \\
\vdots \\
u_{\max }
\end{array}\right] \in \mathbb{R}^{N \cdot m}, \quad U_{\mathrm{MIN}}=\left[\begin{array}{c}
u_{\min } \\
\vdots \\
u_{\min }
\end{array}\right] \in \mathbb{R}^{N \cdot m}
$$

On the other hand, it is also possible to put physical constraints on how fast the inputs can change. The input rate of movement is the amount of control signal that changes when the iteration moves from sample $k-1$ to $k$ and therefore is called $\Delta u_{k}$. The constraints can be directly written as

$$
U_{\mathrm{MIN}}^{d} \leq \Delta u_{k} \leq U_{\mathrm{MAX}}^{d}, \quad \forall k \in\{t, \ldots, t+N-1\}
$$

where

$$
\left.\left.U_{\mathrm{MAX}}^{d}=\left[\begin{array}{c}
u_{\max }^{d} \\
\vdots \\
u_{\max }^{d}
\end{array}\right]\right\} \in \mathbb{R}^{N \cdot m} \text { and } \quad U_{\mathrm{MIN}}^{d}=\left[\begin{array}{c}
u_{\min }^{d} \\
\vdots \\
u_{\min }^{d}
\end{array}\right]\right\} \in \mathbb{R}^{N \cdot m}
$$

which implies

$$
\left[\begin{array}{c}
I_{N \cdot m} \\
-I_{N \cdot m}
\end{array}\right] \Delta u \leq\left[\begin{array}{c}
U_{\mathrm{MAX}}^{d} \\
-U_{\mathrm{MIN}}^{d}
\end{array}\right]
$$

Assuming that a full measurement of the state $x_{t}$ is available at the current time $t$, the following finitehorizon optimal tracking problem is solved at every time instant $t$ to find the optimal control sequence $\Delta u^{\star}$ :

$$
\begin{array}{ll}
\underset{\Delta u}{\operatorname{minimise}} & \frac{1}{2} \Delta u^{T} H \Delta u+\left[\begin{array}{ll}
\tilde{x}_{t}^{T} & r^{T}
\end{array}\right] F \Delta u \\
\text { subject to } & \left\{\begin{array}{l}
\Omega_{1} \\
-\Omega_{1}
\end{array}\right] \Delta u \leq\left[\begin{array}{l}
U_{\mathrm{MAX}} \\
-U_{\mathrm{MIN}}
\end{array}\right]-\left[\begin{array}{c}
\tilde{I}_{m} \\
\\
-\tilde{I}_{m}
\end{array}\right] u_{t-1} \\
{\left[\begin{array}{c}
I_{N \cdot m} \\
-I_{N \cdot m}
\end{array}\right] \Delta u \leq\left[\begin{array}{c}
U_{\mathrm{MAX}}^{d} \\
-U_{\mathrm{MIN}}^{d}
\end{array}\right]}
\end{array}
$$

Once a feasible solution $\Delta u^{\star}$ to the quadratic optimisation problem (51) is obtained, only the first $m$ entries of the signal is used as a control signal for the system 30 . Thus, the control signal at time $t$ can be obtained 


$$
u_{t}=u_{t-1}+\Delta u_{t}
$$

The satisfaction of the predictive control is highly influenced by the choice of the sampling period, $T_{s}$, prediction horizon $N$ and the control horizon $N_{u}$. A recommended practice is to choose $T_{s}$ between $10 \%$ and $25 \%$ of the minimum desired closed-loop response time. Qualitatively, as $T_{s}$ decreases, the satisfaction of the attenuation of the disturbance $w$ usually improves and then does not change much. But, as $T_{s}$ becomes small, the computational effort increases dramatically due to the need for an increase in $N$. Thus, the optimal choice for $T_{s}$ is a trade-off between performance and computational effort.

In MPC, the choice of the prediction horizon, $N$ is also an important consideration. It is the number of predicted future time steps and shows how far the controller predicts into the future. If one chooses to hold the prediction horizon duration $\left(N \times T_{s}\right)$ constant, $N$ must vary inversely with $T_{s}$. As can be seen above, many array sizes are proportional to $N$. Thus, as $N$ increases, the controller memory requirements and quadratic programming solution time increase.

Another design parameter is the control horizon, $N_{u}$. It is the number of non-zero control actions that can be used to keep the system's output in the predicted horizon. Each control move in the control horizon can be thought of as a free variable that needs to be computed by the optimizer. However, it is not a reasonable approach to set $N_{u}=N$, since only the first few steps are dominant on the response of the system. Hence, it is a common practice to keep $N_{u}$ as small as possible and set $u_{k}=0$ for $N_{u}+1 \leq k \leq N$ since, smaller the control horizon, the fewer the computations we have.

In this work, based on the dynamics of the open-loop system, the sampling period is chosen as $T_{s}=0.01$ seconds. Also, through a trial and error approach, it is seen that the best performance is obtained for the design parameters $N=150$ and $N_{u}=2$.

In this note, when $F_{n}=0.5$, using a Zero Order Hold type discretization, one can obtain the system matrices as follows:

$$
\begin{gathered}
\mathcal{A}=\left[\begin{array}{cccc}
0.9998 & 0.0001 & 0.0100 & -0.0003 \\
0.0000 & 0.9998 & 0.0000 & 0.0100 \\
-0.0378 & 0.0122 & 0.9971 & -0.0669 \\
0.0002 & -0.0401 & 0.0002 & 0.9980
\end{array}\right], \quad \mathcal{B}_{w}=10^{-4} \times\left[\begin{array}{cc}
0.0012 & 0.0000 \\
0.0000 & 0.0000 \\
0.2439 & -0.0032 \\
-0.0032 & 0.0024
\end{array}\right] \\
\mathcal{B}=\left[\begin{array}{cc}
0 & 0 \\
0 & 0 \\
-0.0078 & -0.0048 \\
0.0012 & -0.0009
\end{array}\right], \quad \mathcal{C}=\left[\begin{array}{cccc}
0 & 1 & 0 & 0 \\
0 & 0 & 0 & 1
\end{array}\right]
\end{gathered}
$$

For $F_{n}=0.4$, we have the same system matrices given in (54) except 


$$
\mathcal{A}=\left[\begin{array}{cccc}
0.9998 & 0.0001 & 0.0100 & -0.0003 \\
0.0000 & 0.9998 & 0.0000 & 0.0100 \\
-0.0378 & 0.0167 & 0.9969 & -0.0656 \\
0.0002 & -0.0402 & 0.0002 & 0.9972
\end{array}\right], \quad \mathcal{B}=\left[\begin{array}{cc}
0 & 0 \\
0 & 0 \\
-0.0050 & -0.0031 \\
0.0008 & -0.0006
\end{array}\right]
$$

On the other hand, during the design of MPC, weighting matrices of the objective function are chosen as follows: $S=\operatorname{diag}\{100,100\}, Q=\operatorname{diag}\{1,1\}$ and $R=\operatorname{diag}\{0.1,0.1\}$ both for $F_{n}=0.4$ and $F_{n}=0.5$. Finally, the reference trajectory is chosen to be $r=0 \in \mathbb{R}^{300}$ for very iteration $k$.

\section{Discrete-time $\mathcal{H}_{\infty}$ state-feedback control for systems having magnitude and rate-saturated actuators}

In this section, to demonstrate the efficiency of the proposed MPC strategy and to provide a fair comparison with MPC approach, derivation of a novel optimal state-feedback $\mathcal{H}_{\infty}$ controller for discrete-time systems having amplitude and rate limited actuators is considered.

Assume that the vertical motions of a ship are governed by the difference equations (30). Also, assume that the system is subject to control constraints defined by $(31)$ and $(32)$. Note that the control action can be modelled in state-space as

$$
u_{k}=I_{m} u_{k-1}+I_{m} \Delta u_{k}
$$

Then, the augmented state-space system can be written as

$$
\begin{aligned}
\bar{x}_{k+1} & =\underbrace{\left[\begin{array}{cc}
\mathcal{A} & \mathcal{B} \\
0_{m \times n} & I_{m}
\end{array}\right]}_{\mathbb{A}} \underbrace{\left[\begin{array}{c}
x_{k} \\
u_{k-1}
\end{array}\right]}_{\bar{x}_{k}}+\underbrace{\left[\begin{array}{c}
\mathcal{B} \\
I_{m}
\end{array}\right]}_{\mathbb{B}} \Delta u_{k}+\underbrace{\left[\begin{array}{c}
\mathcal{B}_{w} \\
0_{m \times s}
\end{array}\right]}_{\mathbb{B}_{w}} w_{k} \\
y_{k} & =\underbrace{\left[\begin{array}{cc}
\mathcal{C} & 0_{p \times m}
\end{array}\right]}_{\mathbb{C}} \bar{x}_{k}
\end{aligned}
$$

Denoting the transfer function from $w$ to $y$ by $\mathcal{T}_{w y}$, the following theorem provides an optimal static statefeedback $\mathcal{H}_{\infty}$ control law for the augmented system $(56)$.

Theorem 1. There exists a static state-feedback controller in the form of

$$
u_{k}=u_{k-1}+\underbrace{\mathcal{M} \mathcal{X}^{-1}}_{\mathcal{K}} \bar{x}_{k}
$$


such that the inequality $\left\|\mathcal{T}_{w y}\right\|_{\infty}<\gamma$ holds if and only if there exist a symmetric matrix $\mathcal{X}$, and a matrix $\mathcal{M}$ of appropriate dimensions such that the following Linear Matrix Inequalities(LMIs) hold:

$$
\left[\begin{array}{cccc}
\mathcal{X} & 0 & \star & \star \\
0 & \gamma I_{s} & \star & 0 \\
\mathbb{A} \mathcal{X}+\mathbb{B} \mathcal{M} & \mathbb{B}_{w} & \mathcal{X} & 0 \\
\mathbb{C} \mathcal{X} & 0 & 0 & \gamma I_{p}
\end{array}\right] \succeq 0
$$

and

$$
\left[\begin{array}{cc}
\mathcal{X} & \mathcal{M}_{i}^{T} \\
\mathcal{M}_{i} & \left(u_{i, \max }^{d}\right)^{2}
\end{array}\right] \succeq 0, \quad\left[\begin{array}{cc}
\mathcal{X} & \mathcal{X} L_{n+i}^{T} \\
\star & \left(u_{i, \max }-u_{i, \text { max }}^{d}\right)^{2}
\end{array}\right] \succeq 0
$$

for all $i=1, \ldots, m$ where $\mathcal{M}_{i}$ is the $i$-th row of $\mathcal{M}$ and $L_{n+i}$ is a row vector whose $(n+i)$-th entry is 1 and all other entries are 0 . Finally, $u_{i, \max }$ is the $i$-th row of the vector $u_{\max }$ and $u_{i, \max }^{d}$ is the $i$-th row of the vector $u_{\max }^{d}$.

Proof. Consider a Lyapunov functional of the form $V_{k}=\bar{x}_{k}^{T} \mathcal{X}^{-1} \bar{x}_{k}$ at time instant $k$ along the system trajectory (56). Assume that the augmented system is controlled by a control signal of the form $\Delta u_{k}=\mathcal{K} \bar{x}_{k}$. It is easy to show that if

$$
V_{k+1}-V_{k}+\frac{1}{\gamma} y_{k}^{T} y_{k}-\gamma w_{k}^{T} w_{k} \leq 0
$$

then $\left\|\mathcal{T}_{w y}\right\|_{\infty} \triangleq \sup _{w \neq 0}\|y\|_{2}<\gamma \|_{2}<$. According to Bounded-Real Lemma [26], this inequality is equivalent to

$$
\left[\begin{array}{cccc}
\mathcal{X}^{-1} & 0 & \star & \star \\
0 & \gamma I_{s} & \star & 0 \\
\mathcal{X}^{-1} \mathbb{A}+\mathcal{X}^{-1} \mathbb{B} \mathcal{K} & \mathcal{X}^{-1} \mathbb{B}_{w} & \mathcal{X}^{-1} & 0 \\
\mathbb{C} & 0 & 0 & \gamma I_{p}
\end{array}\right] \succeq 0
$$

Applying a congruence transformation by pre- and post multiplying 61 with $\operatorname{diag}\{\mathcal{X}, I, \mathcal{X}, I\}$ and defining $\mathcal{M} \triangleq \mathcal{K} \mathcal{X}$ yields $[58$.

On the other hand, let $\Delta u_{i, k}$ denotes the $i$-th entry of the rate-of-control signal at time instant $k$. From the slew-rate requirements of the control signal, $\left|\Delta u_{i, k}\right| \leq u_{i \text {,max }}^{d}$ must to be satisfied for all $k \geq 0$ and $i=1, \ldots, m$. Note that this inequality is equivalent to $\Delta u_{i, k}^{2} \leq\left(u_{i, \max }^{d}\right)^{2}$ which can be further written as

$$
\bar{x}_{k}^{T} \mathcal{K}_{i}^{T} \mathcal{K}_{i} \bar{x}_{k} \leq\left(u_{i, \max }^{d}\right)^{2}, \quad i=1, \ldots, m
$$

where $\mathcal{K}_{i}$ stands for the $i-$ th row of the feedback matrix $\mathcal{K}$. Rate constraints can be satisfied if the ellipsoid

$$
\operatorname{Elp}\left(\frac{\mathcal{K}_{i}^{T} \mathcal{K}_{i}}{\left(u_{i, \max }^{d}\right)^{2}}\right) \triangleq \bar{x}_{k}^{T} \frac{\mathcal{K}_{i}^{T} \mathcal{K}_{i}}{\left(u_{i, \max }^{d}\right)^{2}} \bar{x}_{k} \leq 1
$$


contains the closed-loop system's energy ellipsoid $\bar{x}_{k}^{T} \mathcal{X}^{-1} \bar{x}_{k} \leq 1$ for all $k \geq 0$. From the theory of ellipsoids 26, this can only happen when

$$
\frac{\mathcal{K}_{i}^{T} \mathcal{K}_{i}}{\left(u_{i, \max }^{d}\right)^{2}} \preceq \mathcal{X}^{-1}, \quad i=1, \ldots, m
$$

Pre- and post multiplying both sides of the 64 by $\mathcal{X}$ and using the definition $\mathcal{K} \mathcal{X}=\mathcal{M}$ yields

$$
\frac{\mathcal{M}_{i}^{T} \mathcal{M}_{i}}{\left(u_{i, \max }^{d}\right)^{2}} \preceq \mathcal{X}, \quad i=1, \ldots, m
$$

Finally, using Schur complement formula [26] on (65) gives the first LMI in (59).

Similarly, let us denote the $i$-th entry of the control vector $u_{k}$ by $u_{i, k}$. The control signal should satisfy $\left|u_{i, k}\right| \leq u_{i, \max }$ for all $k \geq 0$ and $i=1, \ldots, m$ which is equivalent to $u_{i, k}^{2} \leq\left(u_{i, \max }\right)^{2}$. However, since the control system do not have access to $u_{k}$ at sampling time $k$, but $u_{k-1}$, one can consider a bound $\left|u_{i, k-1}\right| \leq u_{i, \max }-u_{i, \max }^{d}$ for all $k \geq 0$ and $i=1, \ldots, m$. This can be further described as an ellipsoid of the form

$$
\operatorname{Elp}\left(\frac{L_{n+i}^{T} L_{n+i}}{\left(u_{i, \max }-u_{i, \max }^{d}\right)^{2}}\right) \triangleq \bar{x}_{k}^{T} \frac{L_{n+i}^{T} L_{n+i}}{\left(u_{i, \max }-u_{i, \max }^{d}\right)^{2}} \bar{x}_{k} \leq 1
$$

So, magnitude constraints on the control signal can be satisfied at all times if

$$
\operatorname{Elp}\left(\frac{L_{n+i}^{T} L_{n+i}}{\left(u_{i, \max }-u_{i, \max }^{d}\right)^{2}}\right) \preceq \mathcal{X}^{-1}, \quad i=1, \ldots, m
$$

Pre- and post multiplying both sides of the [67] by $\mathcal{X}$ and using Schur complement formula [26] yields the second LMI given in (59). This concludes the proof.

\section{Simulation Study}

In this section, the results of the simulation studies which were performed for mitigating the vertical accelerations of the passenger ship in irregular head waves having Froude number $F_{n}=\frac{V}{\sqrt{g L_{W L}}}=0.40$ and 0.50 are presented with the help of several tables and graphs. The optimisation problem (51) is solved in real time for a suitable MPC using the system matrices given in 54). All computations are accomplished using MATLAB along with quadprog solver by taking all initial conditions equal to zero. Each simulation representing $H_{s}=0.70,0.88$ and 1.00 metres at $F_{n}=0.40$ and $F_{n}=0.50$ last 100 seconds. In order to compare the performance of the proposed MPC with another optimal control method, discrete time $\mathcal{H}_{\infty}$ state feedback controller has been performed for the modelled passenger ship under consideration of magnitude and rate saturated foils.

During the simulation study, an $\mathcal{H}_{\infty}$ controller gain

$$
\mathcal{K}=\left[\begin{array}{cccccc}
0.0007 & 0.1905 & -0.0024 & -0.0351 & -0.0042 & 0.0035 \\
-0.0020 & -0.1776 & 0.0019 & 0.0102 & 0.0036 & -0.0033
\end{array}\right]
$$


is used for $F_{n}=0.4$, and

$$
\mathcal{K}=\left[\begin{array}{cccccc}
-0.0175 & -3.5550 & -0.0160 & -9.3183 & -0.1187 & 0.0891 \\
0.0173 & 3.9295 & 0.0161 & 9.2341 & 0.1191 & -0.0900
\end{array}\right]
$$

for $F_{n}=0.5$. These controller gains are obtained by solving the LMIs given in (58) and (59) using YALMIP 27] with SeduMi solver in MATLAB. Time-domain comparison of uncontrolled and controlled states (MPC and $\mathcal{H}_{\infty}$ ) and vertical accelerations at the bow, stern and central locations of the ship for $F_{n}=0.40$ are given in Figure 9 - Figure 14 for $H_{s}=0.70,0.88$ and 1.00 metres, respectively.
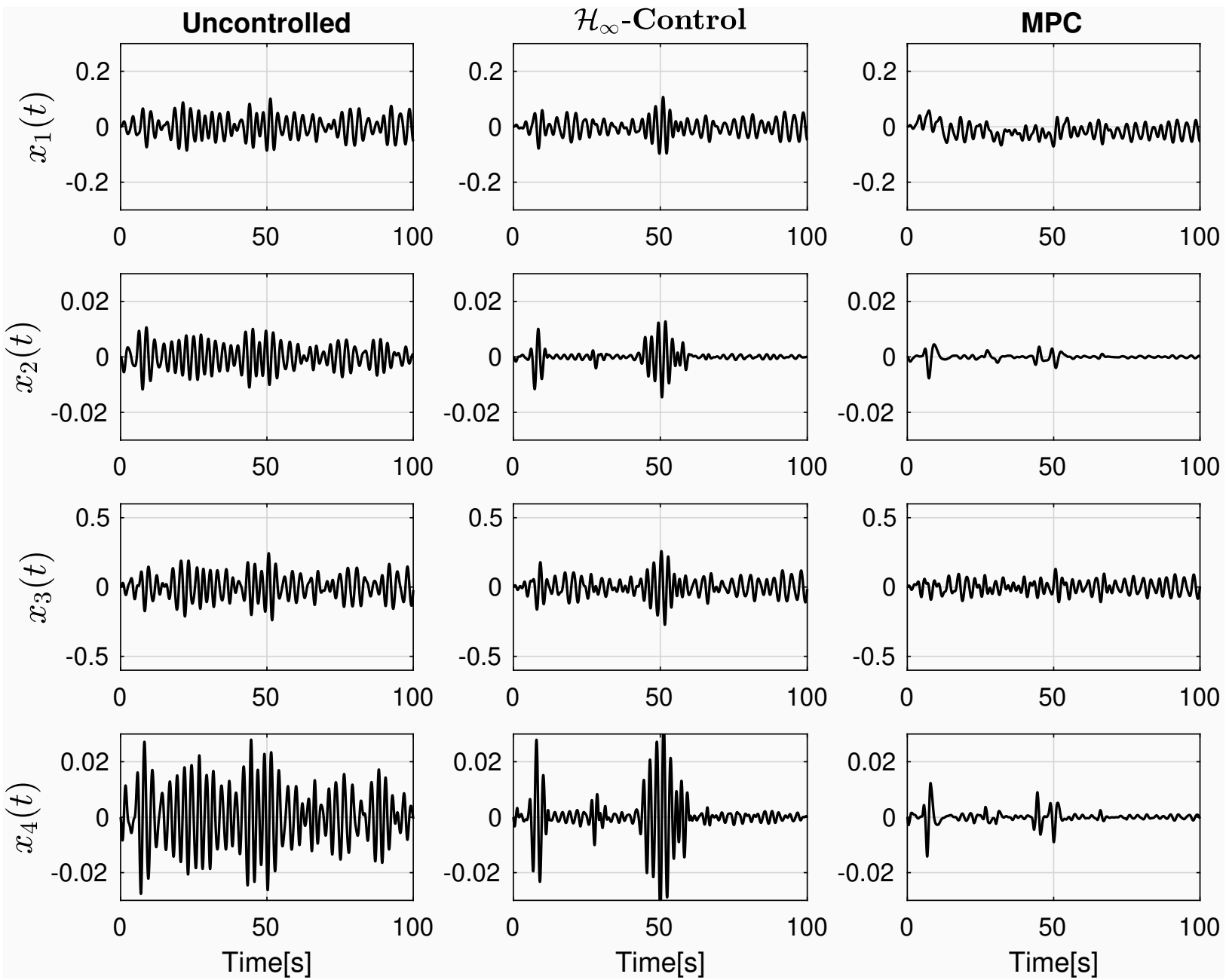

Figure 9: Time histories of uncontrolled and controlled states at $F_{n}=0.40\left(H_{s}=0.70 \mathrm{~m}\right)$.

Similarly, time-domain comparison of uncontrolled and controlled states (MPC and $\mathcal{H}_{\infty}$ ) and vertical accelerations at the bow, stern and central locations of the ship for $F_{n}=0.50$ are provided in Figure 15 Figure 20 for $H_{s}=0.70,0.88$ and 1.00 metres, respectively. Figures 9 Figure 20 reveal that the MPC design 225 is very successful in reducing pitch motion and pitch velocity for two forward ship speeds even in higher 

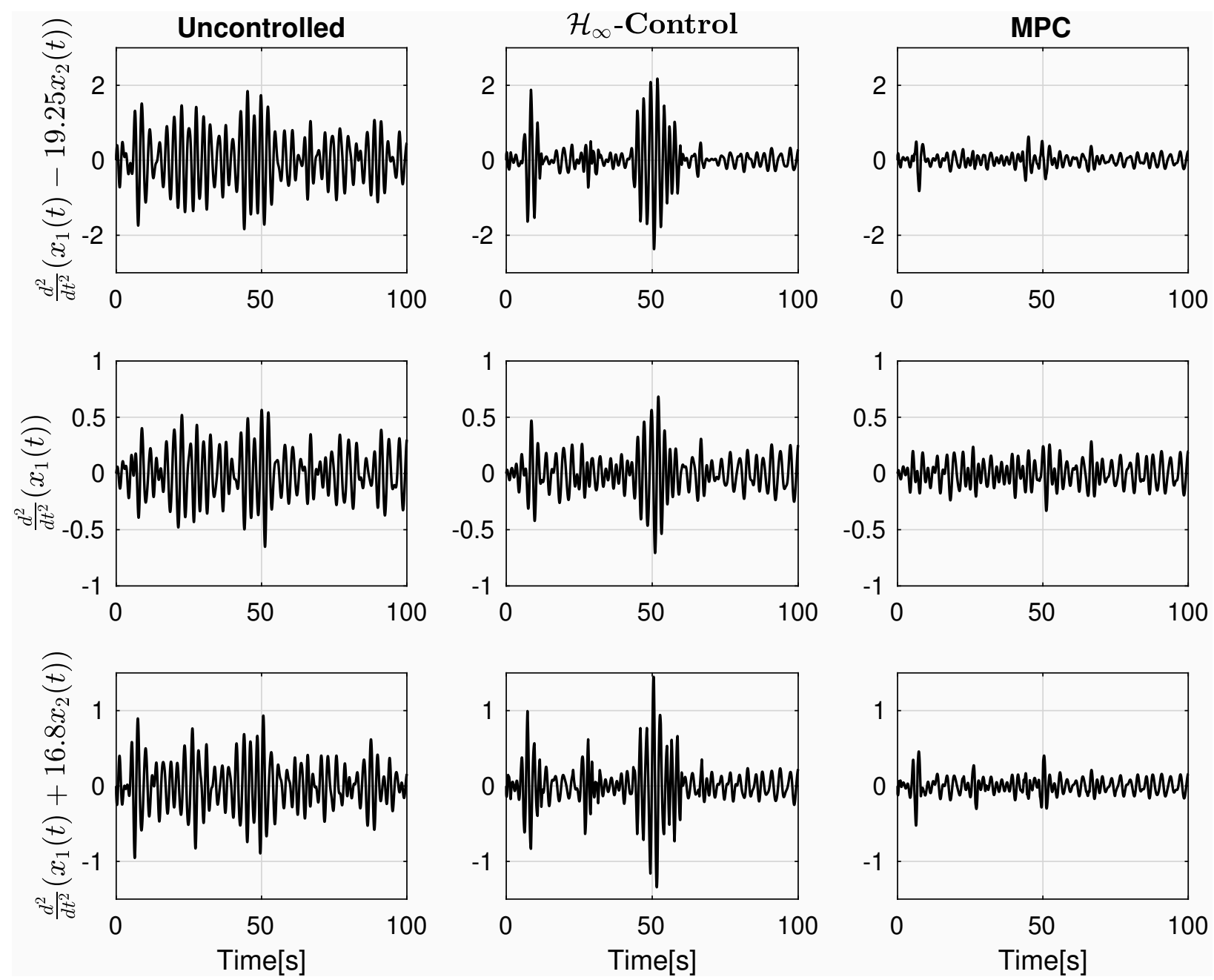

Figure 10: Time histories of uncontrolled and controlled accelerations at $F_{n}=0.40\left(H_{s}=0.70 \mathrm{~m}\right)$.

sea states. In conjunction with the improvements in pitch motion and pitch velocity of the passenger ship, bow and stern accelerations are reduced remarkably as well particularly in $H_{s}=0.70$ metres significant wave height. It is also noted that the attenuations of the heave acceleration are less than the attenuation of the bow and stern accelerations due to the phase difference between the pitch and heave motions. In other words, only heave or pitch motion can be attenuated with a high level of efficiency. As known, the consideration of the seasickness index of the passengers who sit at bow and stern are more important since the motion is larger at bow and stern than center. Therefore, in this study, it is aimed to reduce preferably pitch motion and velocity rather than heave motion and velocity.

On the other hand, the control signals for the real foil angles $\beta(t)$ and their rates $\dot{\beta}(t)$ at $F_{n}=0.40$ 235 are given in Figure 21, Figure 22 and Figure 23 in corresponding sea sates. In the same way, the control signals for the real foil angles $\beta(t)$ and their rates $\dot{\beta}(t)$ at $F_{n}=0.50$ are given in Figure 24, Figure 25 and 

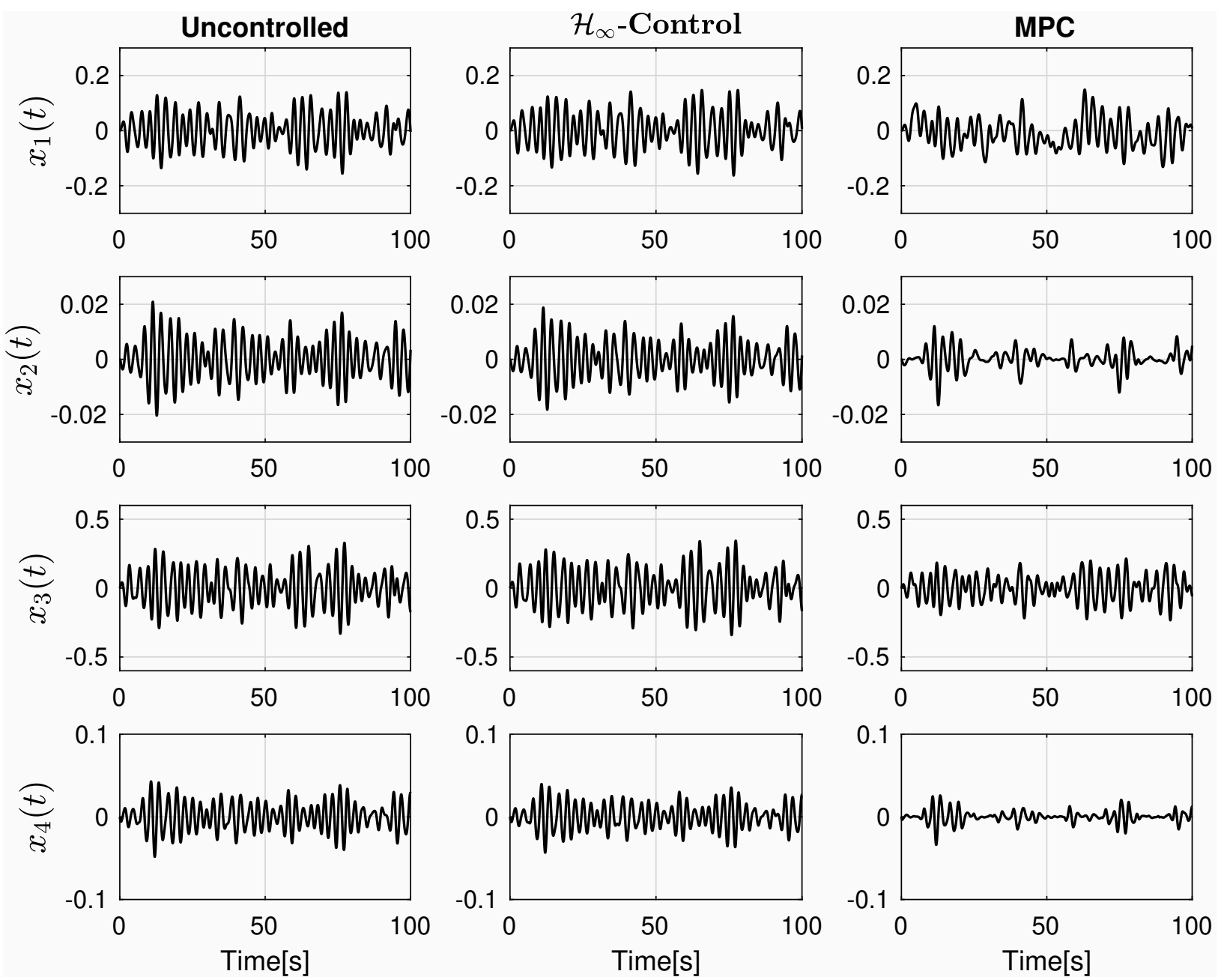

Figure 11: Time histories of uncontrolled and controlled states at $F_{n}=0.40\left(H_{s}=0.88 \mathrm{~m}\right)$.

Figure 26 in corresponding sea sates. As it can be seen from the figures, both the foil angles and their rates are remained within the limits of actuator opening angle (20deg, 20deg) and (20deg/s, 20deg/s) rate of opening angles at all times for $F_{n}=0.40$ and $F_{n}=0.50$.

In Table 3 - Table 5 , the responses at $F_{n}=0.40$ are tabulated with uncontrolled and controlled responses of the passenger ship under the disturbance corresponding to $H_{s}=0.70,0.88$ and 1.00 metres, respectively. In Table 6- Table 8 , the responses at $F_{n}=0.50$ are tabulated with uncontrolled and controlled responses of the passenger ship under the disturbance corresponding to $H_{s}=0.70,0.88$ and 1.00 metres, respectively.

As it is readily seen from Table 3- Table 8, conducted simulation studies show that the designed MPC controller has better performance than $\mathcal{H}_{\infty}$ controller. The underlying reason behind this is, MPC has advantages in a wave attenuation problem with its preview capability of wave disturbances and handling the foil angle and rate of foil angle constraints. Therefore, Table 9 and Table 10 summarise the acceleration 

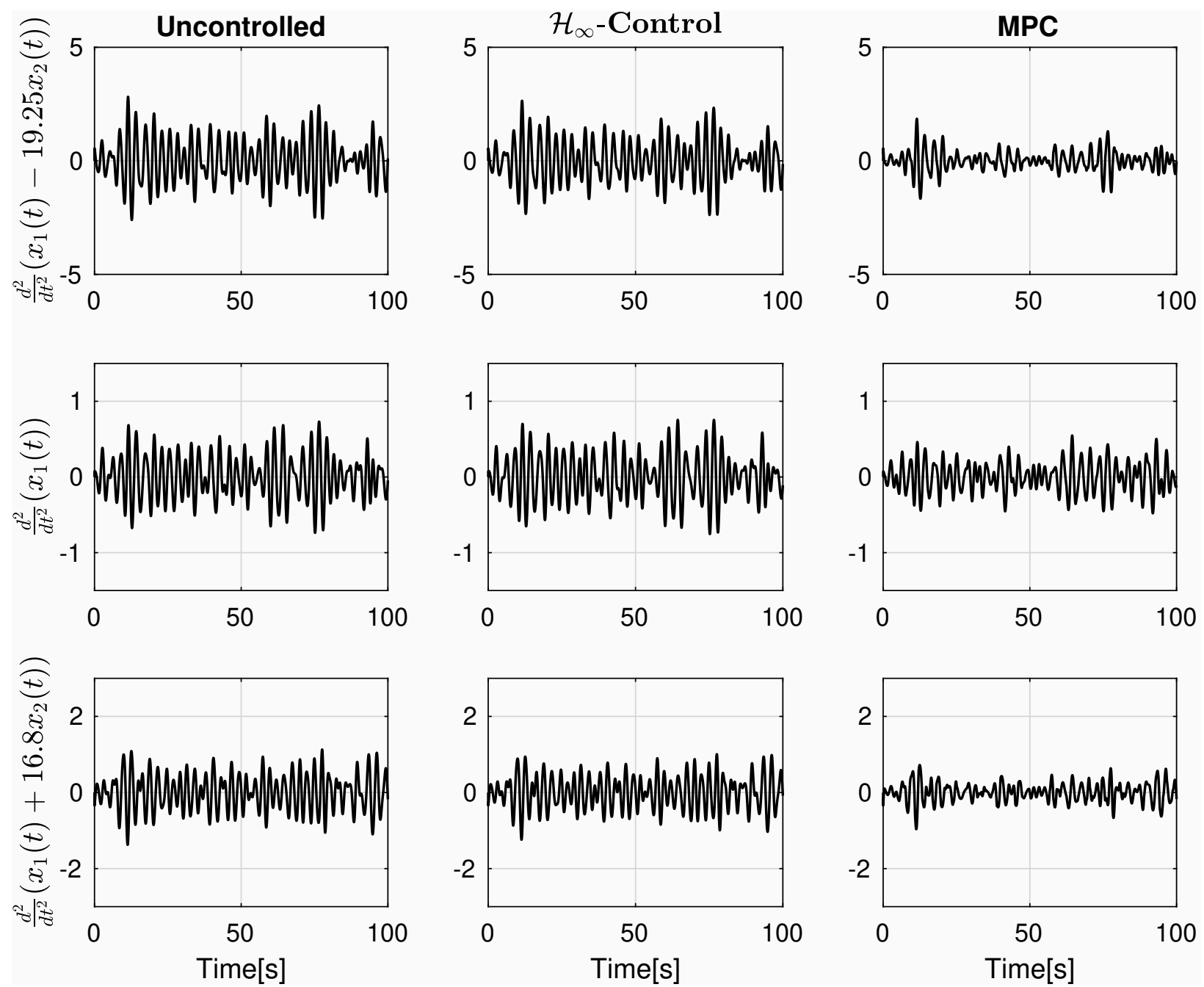

Figure 12: Time histories of uncontrolled and controlled accelerations at $F_{n}=0.40\left(H_{s}=0.88 \mathrm{~m}\right)$.

Table 3: Uncontrolled and controlled vertical responses at $F_{n}=0.40\left(H_{s}=0.70\right.$ metres $)$.

\begin{tabular}{lccc}
\hline \hline & Uncontrolled & Controlled $\left(\mathcal{H}_{\infty}\right)$ & Controlled (MPC) \\
\hline Pitch Motion (rms) (radians) & 0.00425 & 0.00282 & 0.00117 \\
Bow Vertical Acceleration $(\mathrm{rms})\left(\mathrm{m} / \mathrm{s}^{2}\right)$ & 0.6806 & 0.5077 & 0.1695 \\
Stern Vertical Acceleration $(\mathrm{rms})\left(\mathrm{m} / \mathrm{s}^{2}\right)$ & 0.3085 & 0.2878 & 0.1111 \\
CoG Acceleration $(\mathrm{rms})\left(\mathrm{m} / \mathrm{s}^{2}\right)$ & 0.2085 & 0.1719 & 0.1072 \\
\hline
\end{tabular}

reduction capabilities of $\mathrm{MPC}$ for $F_{n}=0.40$ and $F_{n}=0.50$. As seen from Table 9 and Table 10 the designed MPC controller is generally more effective at $F_{n}=0.50$ since the advance speed of the ship affects the control input matrices quadratically. It is interesting to note that the excitation terms are also different at different Froude numbers when the ship is exposed to the same wave spectrum. Therefore, the efficiency 

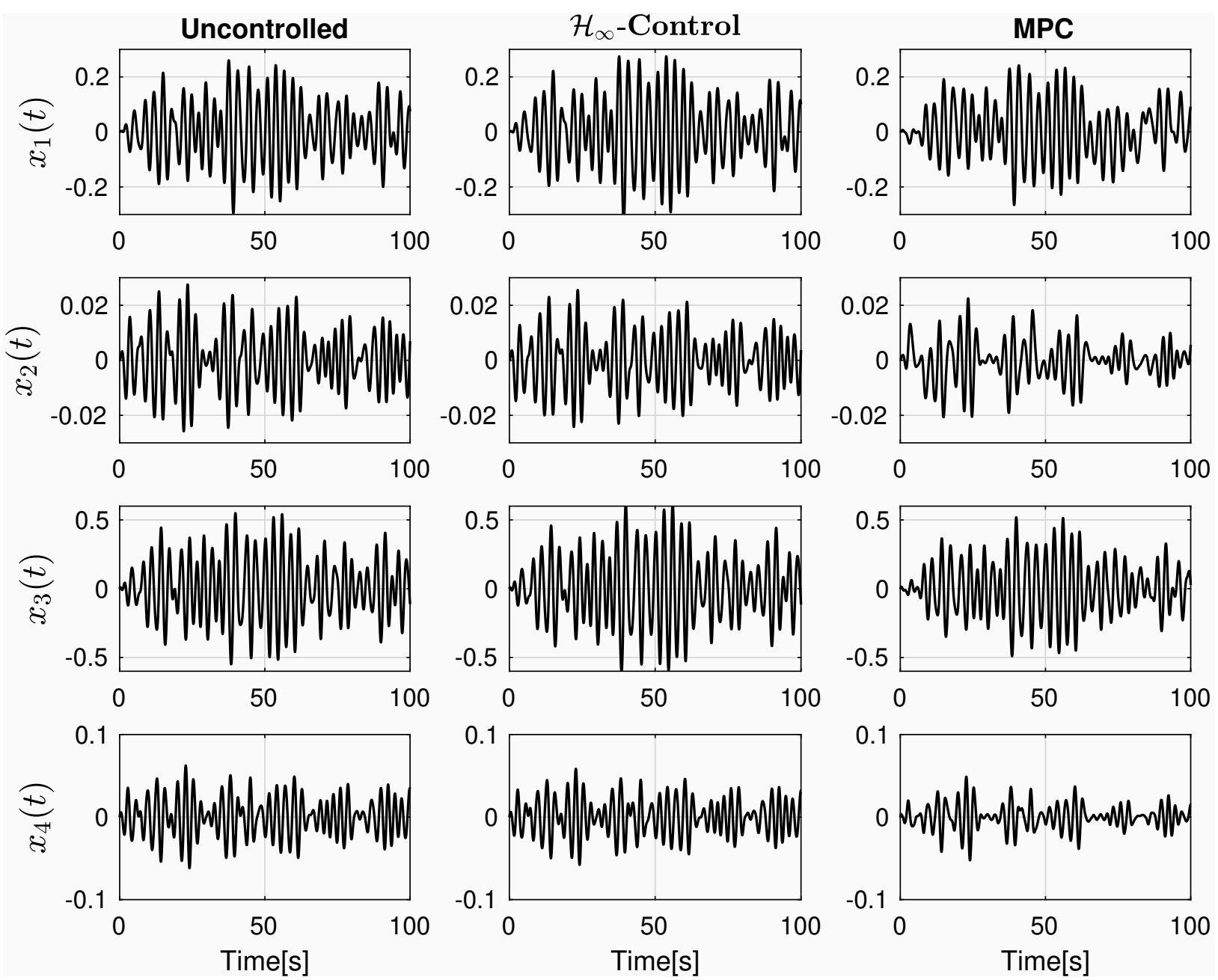

Figure 13: Time histories of uncontrolled and controlled states at $F_{n}=0.40\left(H_{s}=1.00 \mathrm{~m}\right)$.

Table 4: Uncontrolled and controlled vertical responses at $F_{n}=0.40\left(H_{s}=0.88\right.$ metres $)$.

\begin{tabular}{lccc}
\hline \hline & Uncontrolled & Controlled $\left(\mathcal{H}_{\infty}\right)$ & Controlled $(\mathrm{MPC})$ \\
\hline Pitch Motion (rms) (rad.) & 0.00742 & 0.00672 & 0.00356 \\
Bow Vertical Acceleration $(\mathrm{rms})\left(\mathrm{m} / \mathrm{s}^{2}\right)$ & 0.9767 & 0.9162 & 0.4401 \\
Stern Vertical Acceleration $(\mathrm{rms})\left(\mathrm{m} / \mathrm{s}^{2}\right)$ & 0.4809 & 0.4397 & 0.2466 \\
CoG Acceleration $(\mathrm{rms})\left(\mathrm{m} / \mathrm{s}^{2}\right)$ & 0.2985 & 0.3039 & 0.1998 \\
\hline
\end{tabular}

of the controller is also dependent of excitation terms. That is the reason why a direct proportional relation may not set for the motion reductions at $F_{n}=0.40$ and $F_{n}=0.50$.

Ship accelerations, especially the vertical ones, have a great impact on the human body since they may cause motion sickness. The term motion sickness on ships is named as seasickness and it is a health problem 

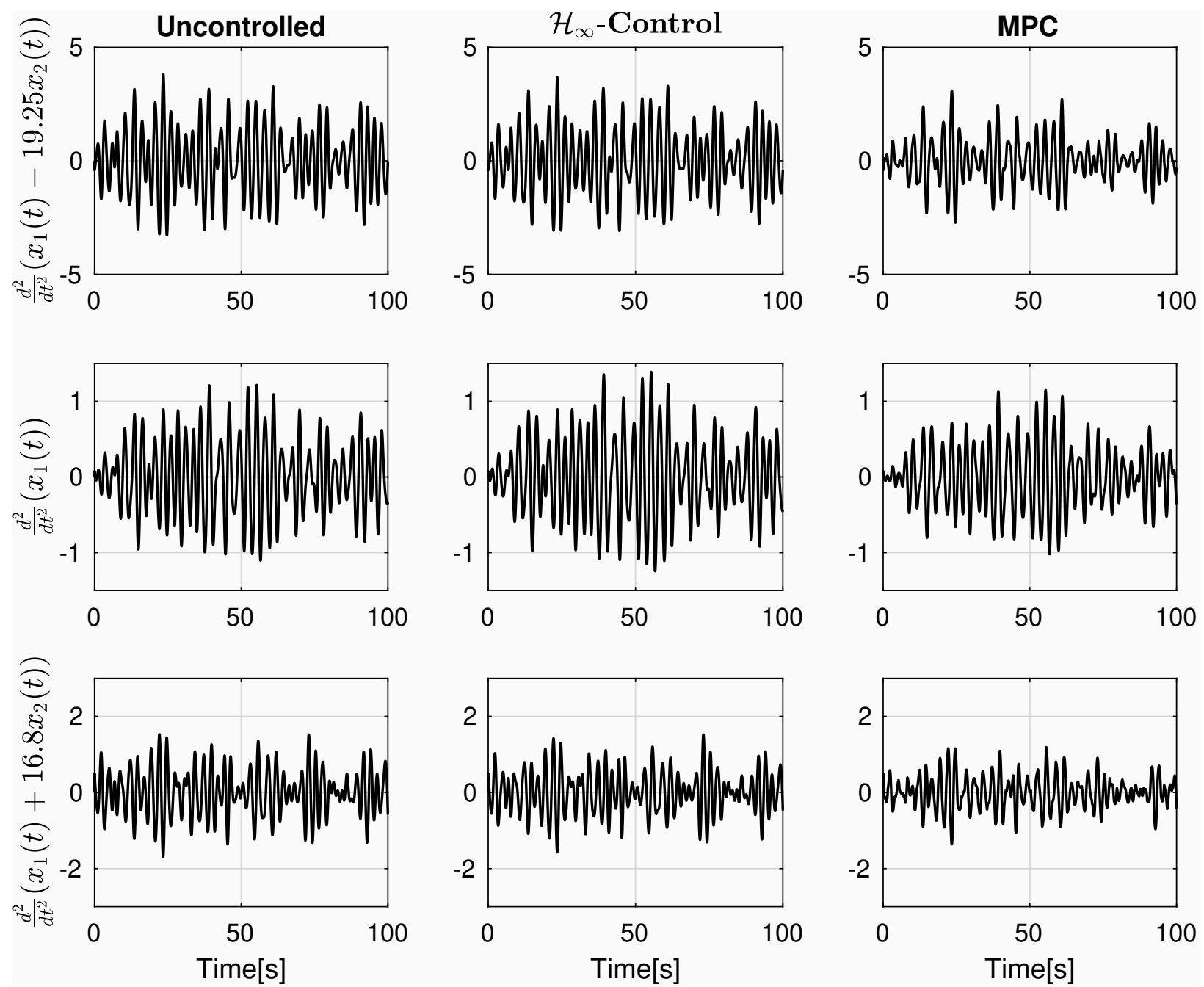

Figure 14: Time histories of uncontrolled and controlled accelerations at $F_{n}=0.40\left(H_{s}=1.00 \mathrm{~m}\right)$.

Table 5: Uncontrolled and controlled vertical responses at $F_{n}=0.40\left(H_{s}=1.00\right.$ metres $)$.

\begin{tabular}{lccc}
\hline \hline & Uncontrolled & Controlled $\left(\mathcal{H}_{\infty}\right)$ & Controlled $(\mathrm{MPC})$ \\
\hline Pitch Motion (rms) (rad.) & 0.01052 & 0.00982 & 0.00708 \\
Bow Vertical Acceleration $(\mathrm{rms})\left(\mathrm{m} / \mathrm{s}^{2}\right)$ & 1.4439 & 1.4127 & 0.9498 \\
Stern Vertical Acceleration $(\mathrm{rms})\left(\mathrm{m} / \mathrm{s}^{2}\right)$ & 0.6079 & 0.5573 & 0.4283 \\
CoG Acceleration $(\mathrm{rms})\left(\mathrm{m} / \mathrm{s}^{2}\right)$ & 0.4926 & 0.53293 & 0.4162 \\
\hline
\end{tabular}

due to ship motions that may result in physical discomfort on the body. Seasickness might cause irregular breathing, nausea, vertigo and vomiting. The Motion Sickness Index (MSI) is commonly used for assessing the likely occurrence of the illness. As discussed in [28, MSI can be expressed as a function of the mean absolute value of vertical acceleration signal and the mean peak frequency of the vertical acceleration signal. 

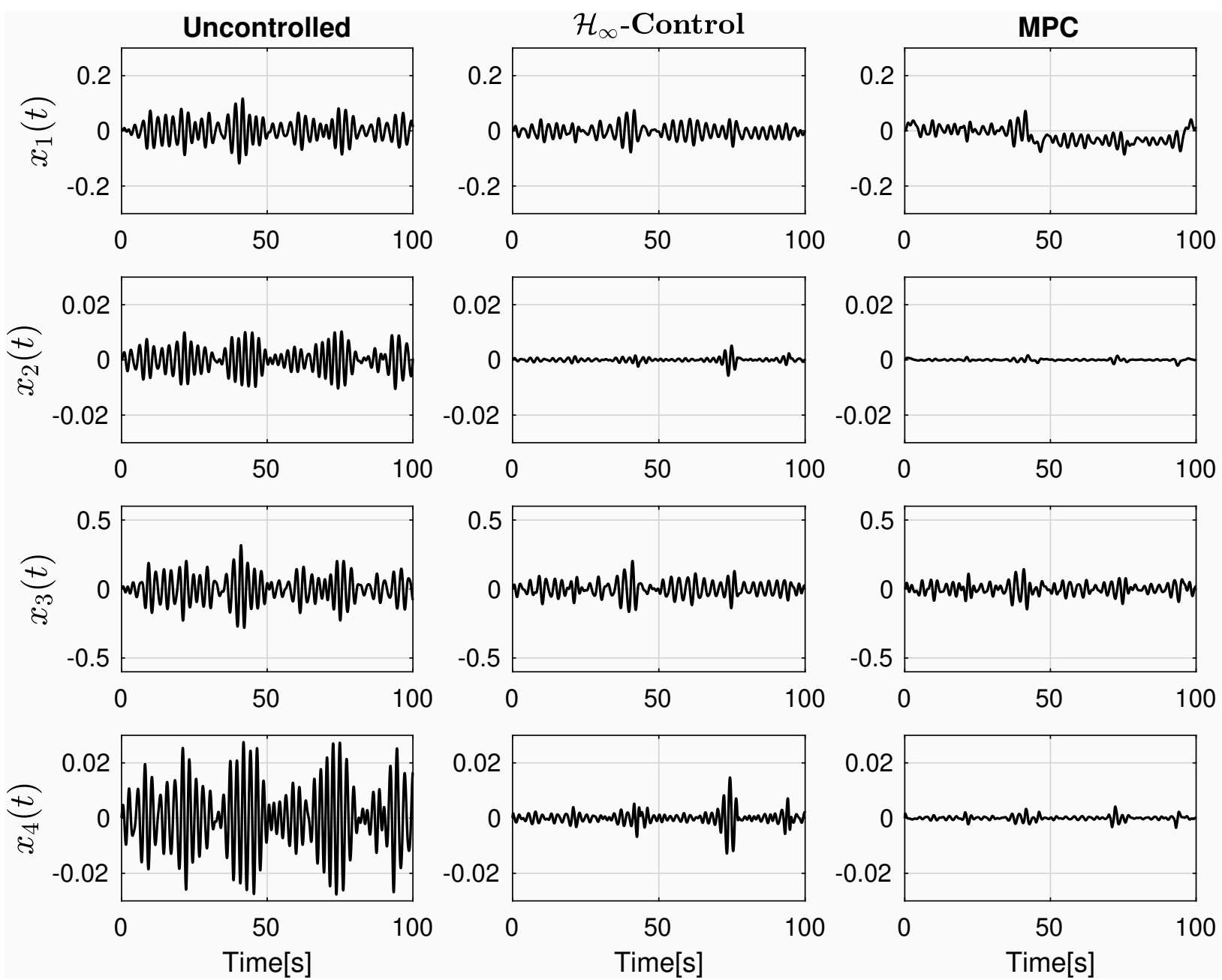

Figure 15: Time histories of uncontrolled and controlled states at $F_{n}=0.50\left(H_{s}=0.70 \mathrm{~m}\right)$.

Table 6: Uncontrolled and controlled vertical responses at $F_{n}=0.50\left(H_{s}=0.70\right.$ metres $)$.

\begin{tabular}{lccc}
\hline \hline & Uncontrolled & Controlled $\left(\mathcal{H}_{\infty}\right)$ & Controlled $(\mathrm{MPC})$ \\
\hline Pitch Motion (rms) (radians) & 0.00426 & 0.00087 & 0.00039 \\
Bow Vertical Acceleration $(\mathrm{rms})\left(\mathrm{m} / \mathrm{s}^{2}\right)$ & 0.7885 & 0.2156 & 0.1389 \\
Stern Vertical Acceleration $(\mathrm{rms})\left(\mathrm{m} / \mathrm{s}^{2}\right)$ & 0.3662 & 0.1552 & 0.0831 \\
CoG Acceleration $(\mathrm{rms})\left(\mathrm{m} / \mathrm{s}^{2}\right)$ & 0.2387 & 0.1307 & 0.1051 \\
\hline
\end{tabular}

Since we have time series of vertical acceleration data for uncontrolled and controlled cases, both values can be obtained easily and finally MSI can be calculated. Table 11 shows the MSI for uncontrolled and controlled (MPC) cases for different locations on the ship and different sea states as regards the two hours cruise $\left(F_{n}=0.50\right)$. Results indicate that a significant decrease in the MSI values are observed in $H_{s}=0.70$ 

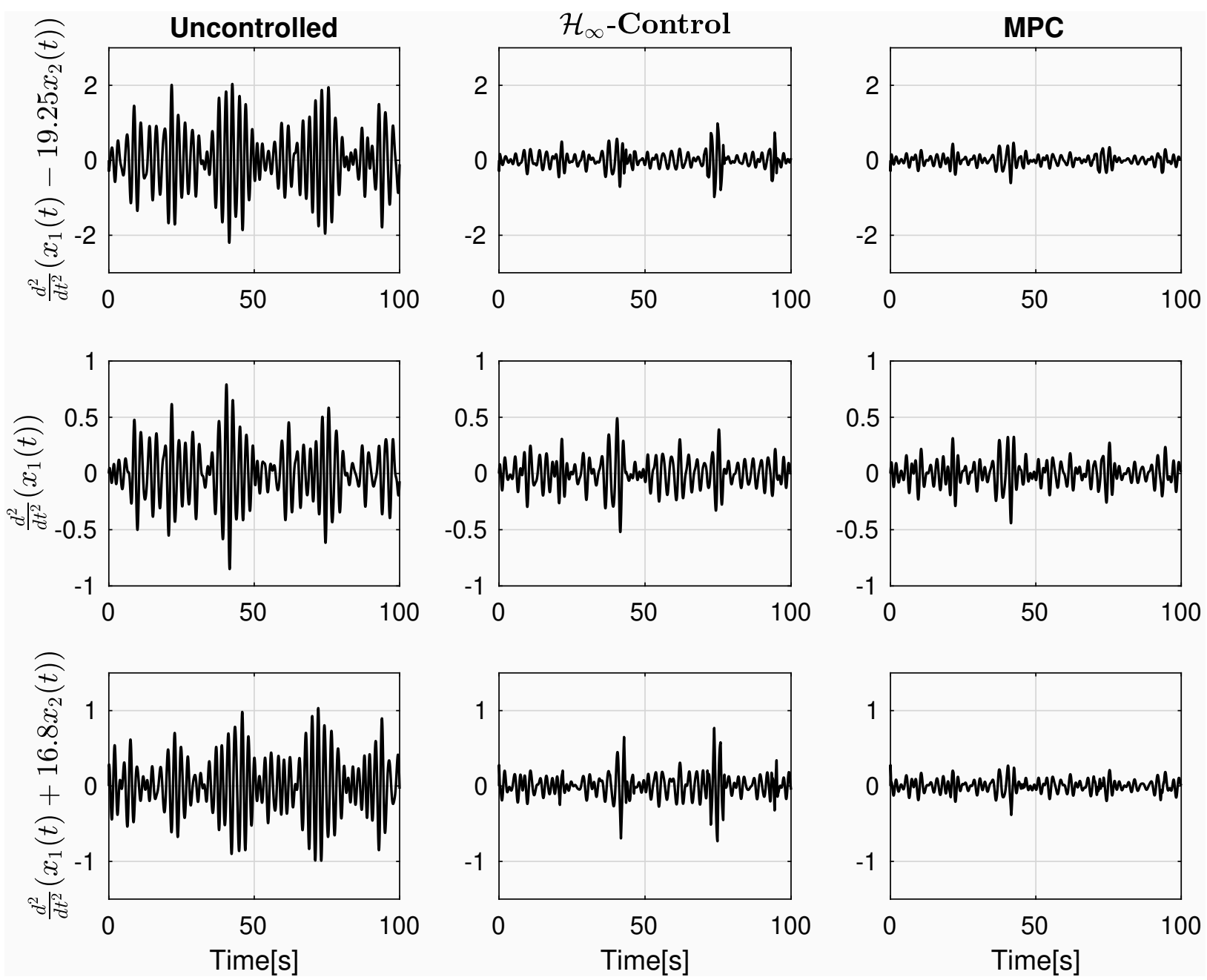

Figure 16: Time histories of uncontrolled and controlled accelerations at $F_{n}=0.50\left(H_{s}=0.70 \mathrm{~m}\right)$.

Table 7: Uncontrolled and controlled vertical responses at $F_{n}=0.50\left(H_{s}=0.88\right.$ metres $)$.

\begin{tabular}{lccc}
\hline \hline & Uncontrolled & Controlled $\left(\mathcal{H}_{\infty}\right)$ & Controlled $(\mathrm{MPC})$ \\
\hline Pitch Motion (rms) (rad.) & 0.00842 & 0.00653 & 0.00459 \\
Bow Vertical Acceleration $(\mathrm{rms})\left(\mathrm{m} / \mathrm{s}^{2}\right)$ & 1.3835 & 1.1374 & 0.8156 \\
Stern Vertical Acceleration $(\mathrm{rms})\left(\mathrm{m} / \mathrm{s}^{2}\right)$ & 0.6663 & 0.5698 & 0.3863 \\
CoG Acceleration $(\mathrm{rms})\left(\mathrm{m} / \mathrm{s}^{2}\right)$ & 0.4083 & 0.3850 & 0.2987 \\
\hline
\end{tabular}

and remarkable improvements are observed even in higher sea states. 

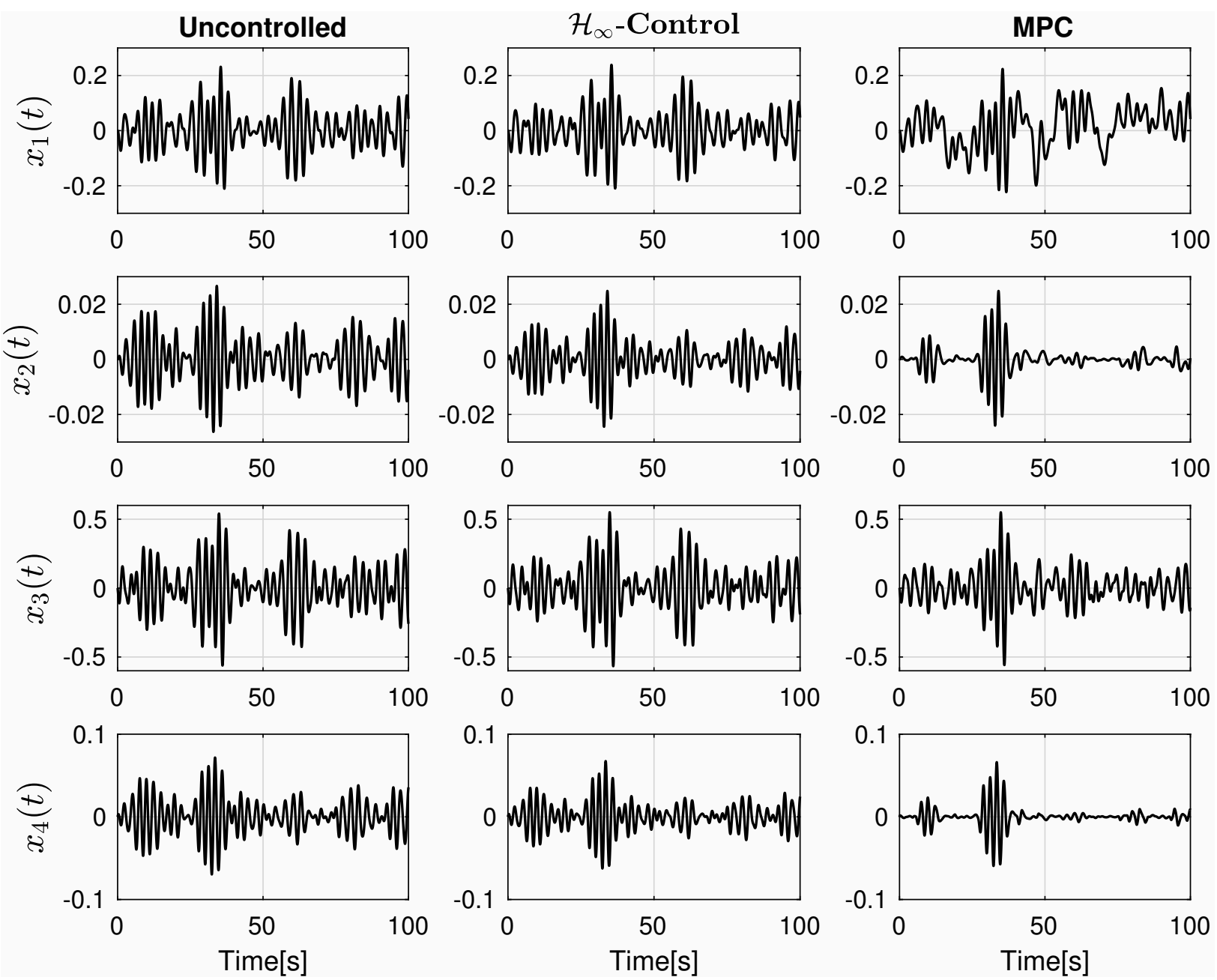

Figure 17: Time histories of uncontrolled and controlled states at $F_{n}=0.50\left(H_{s}=0.88 \mathrm{~m}\right)$.

Table 8: Uncontrolled and controlled vertical responses at $F_{n}=0.50\left(H_{s}=1.00\right.$ metres $)$.

\begin{tabular}{lccc}
\hline \hline & Uncontrolled & Controlled $\left(\mathcal{H}_{\infty}\right)$ & Controlled $(\mathrm{MPC})$ \\
\hline Pitch Motion (rms) (rad.) & 0.01095 & 0.008554 & 0.00663 \\
Bow Vertical Acceleration $(\mathrm{rms})\left(\mathrm{m} / \mathrm{s}^{2}\right)$ & 1.6248 & 1.3636 & 0.9689 \\
Stern Vertical Acceleration $(\mathrm{rms})\left(\mathrm{m} / \mathrm{s}^{2}\right)$ & 0.8348 & 0.7207 & 0.4581 \\
CoG Acceleration $(\mathrm{rms})\left(\mathrm{m} / \mathrm{s}^{2}\right)$ & 0.5045 & 0.5124 & 0.4125 \\
\hline
\end{tabular}

\section{Conclusion}

In this study, it was aimed to reduce the vertical accelerations of a passenger ship which is operating at two different advance speed and in the different level magnitude of head waves. For this reason, Cummins' equation was solved with the time domain identification of fluid memory effects. Firstly, radiation terms 

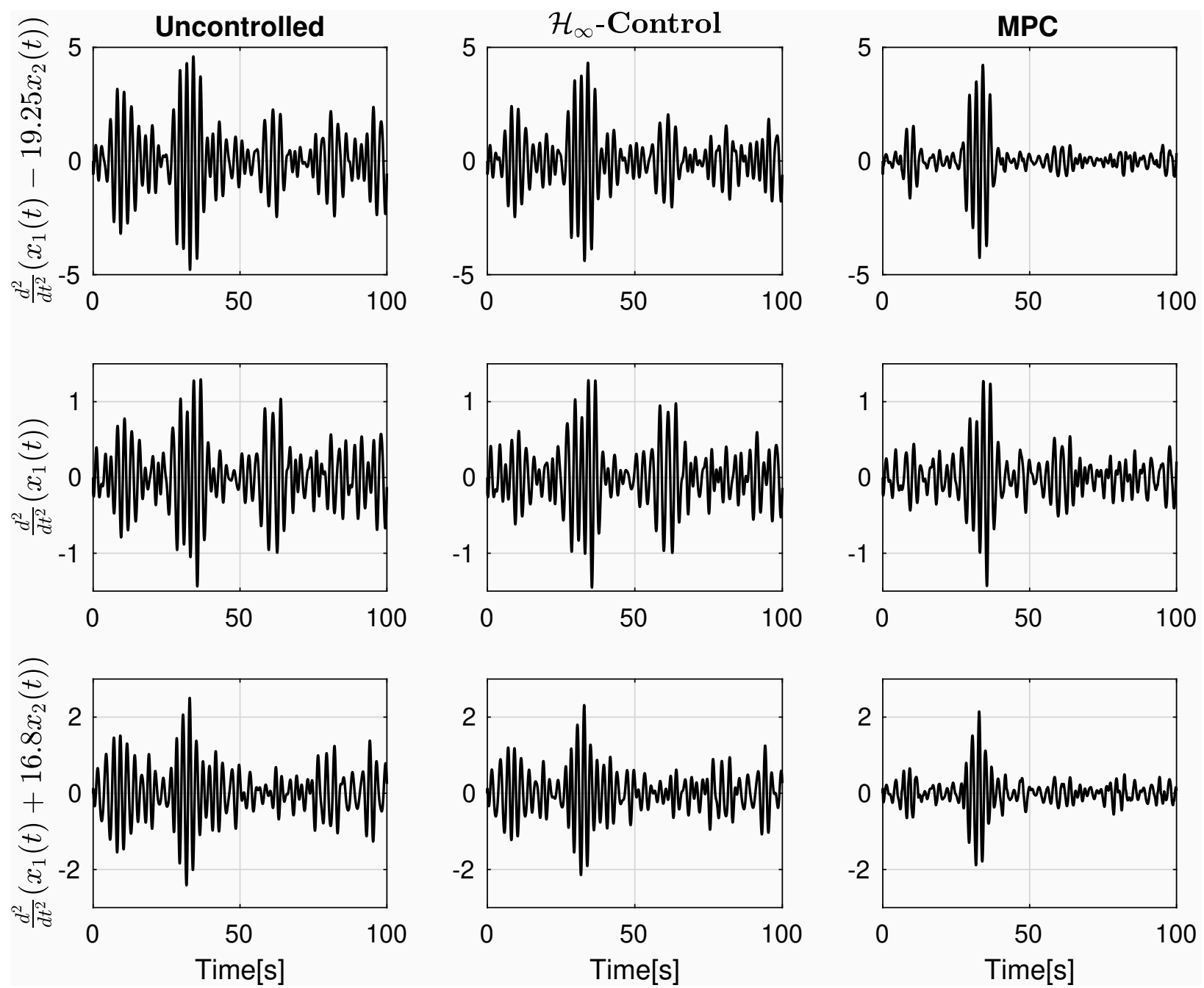

Figure 18: Time histories of uncontrolled and controlled accelerations at $F_{n}=0.50\left(H_{s}=0.88 \mathrm{~m}\right)$.

Table 9: Percentages improvements of accelerations at different sea states $\left(F_{n}=0.40\right)$ by MPC.

\begin{tabular}{lccc}
\hline \hline & $\begin{array}{c}\text { \% Improvement } \\
\text { at } H_{s}=0.70\end{array}$ & $\begin{array}{c}\text { \% Improvement } \\
\text { at } H_{s}=0.88\end{array}$ & $\begin{array}{c}\text { Improvement } \\
\text { at } H_{s}=1.00\end{array}$ \\
\hline Pitch Motion & 72.36 & 51.97 & 32.64 \\
Bow Vertical Acceleration & 75.09 & 54.93 & 34.21 \\
Stern Vertical Acceleration & 63.97 & 48.71 & 29.54 \\
CoG Acceleration & 48.56 & 31.22 & 15.49 \\
\hline
\end{tabular}

were calculated in the frequency domain. By the aid of the information in the frequency domain, all parameters in the Cummins' equation were set for the solution in the time domain. The heave force and pitch moment acting on the ship due to irregular head waves were predicted in the time domain by the 

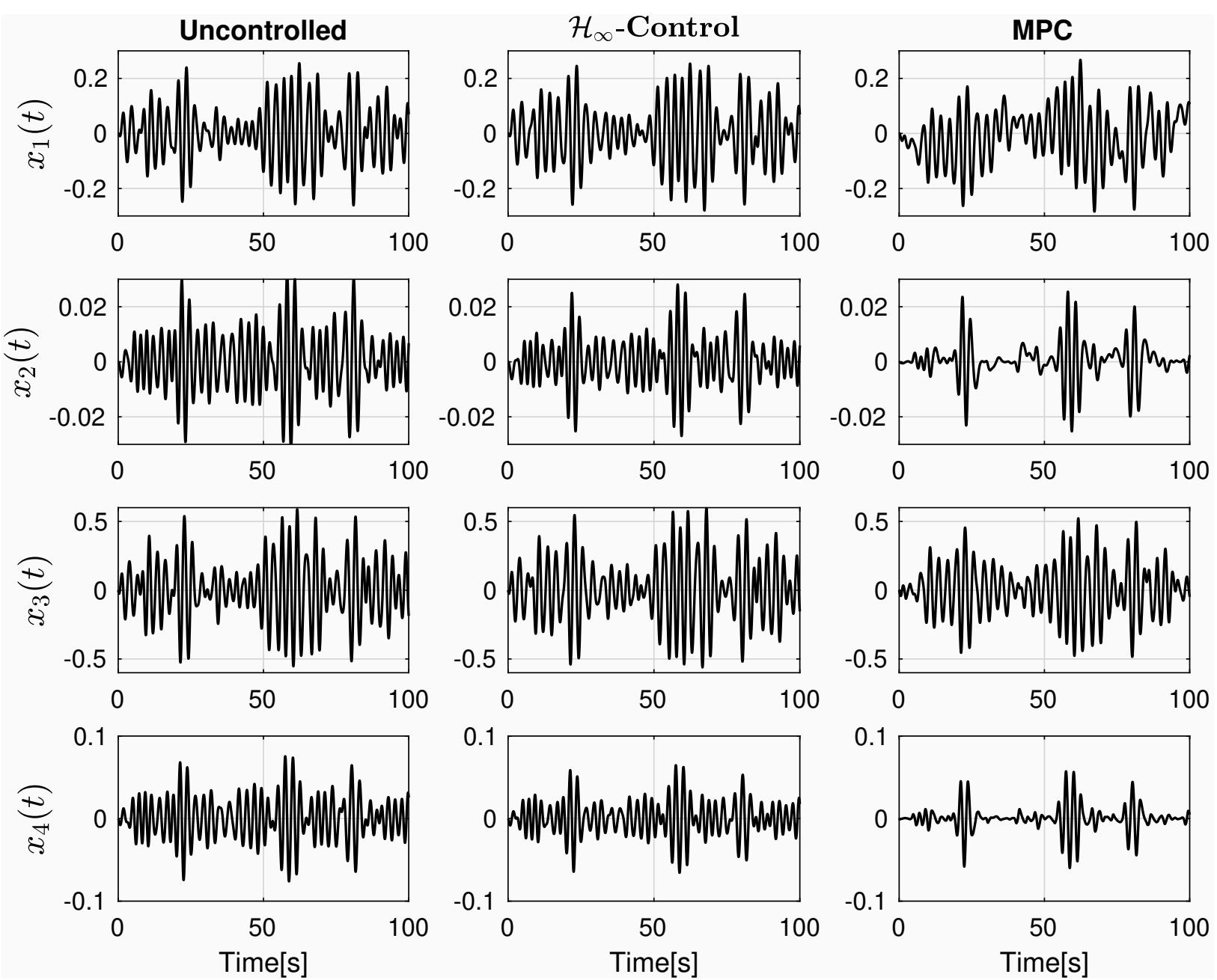

Figure 19: Time histories of uncontrolled and controlled states at $F_{n}=0.50\left(H_{s}=1.00 \mathrm{~m}\right)$.

Table 10: Percentages improvements of accelerations at different sea states $\left(F_{n}=0.50\right)$ by MPC.

$\%$ Improvement \% Improvement \% Improvement

\begin{tabular}{lccc} 
& at $H_{s}=0.70$ & at $H_{s}=0.88$ & at $H_{s}=1.00$ \\
\hline Pitch Motion & 90.67 & 45.47 & 39.42 \\
Bow Vertical Acceleration & 82.37 & 41.04 & 40.36 \\
Stern Vertical Acceleration & 77.32 & 42.01 & 45.12 \\
CoG Acceleration & 55.93 & 26.84 & 18.23 \\
\hline
\end{tabular}

randomization process of the excitation response spectra. Then, an optimal MPC was designed to reduce the vertical accelerations. During operation of the passenger ship, all states were measured with sensors because MPC works with full state feedback procedure. The designed MPC focused on reducing pitch motion 

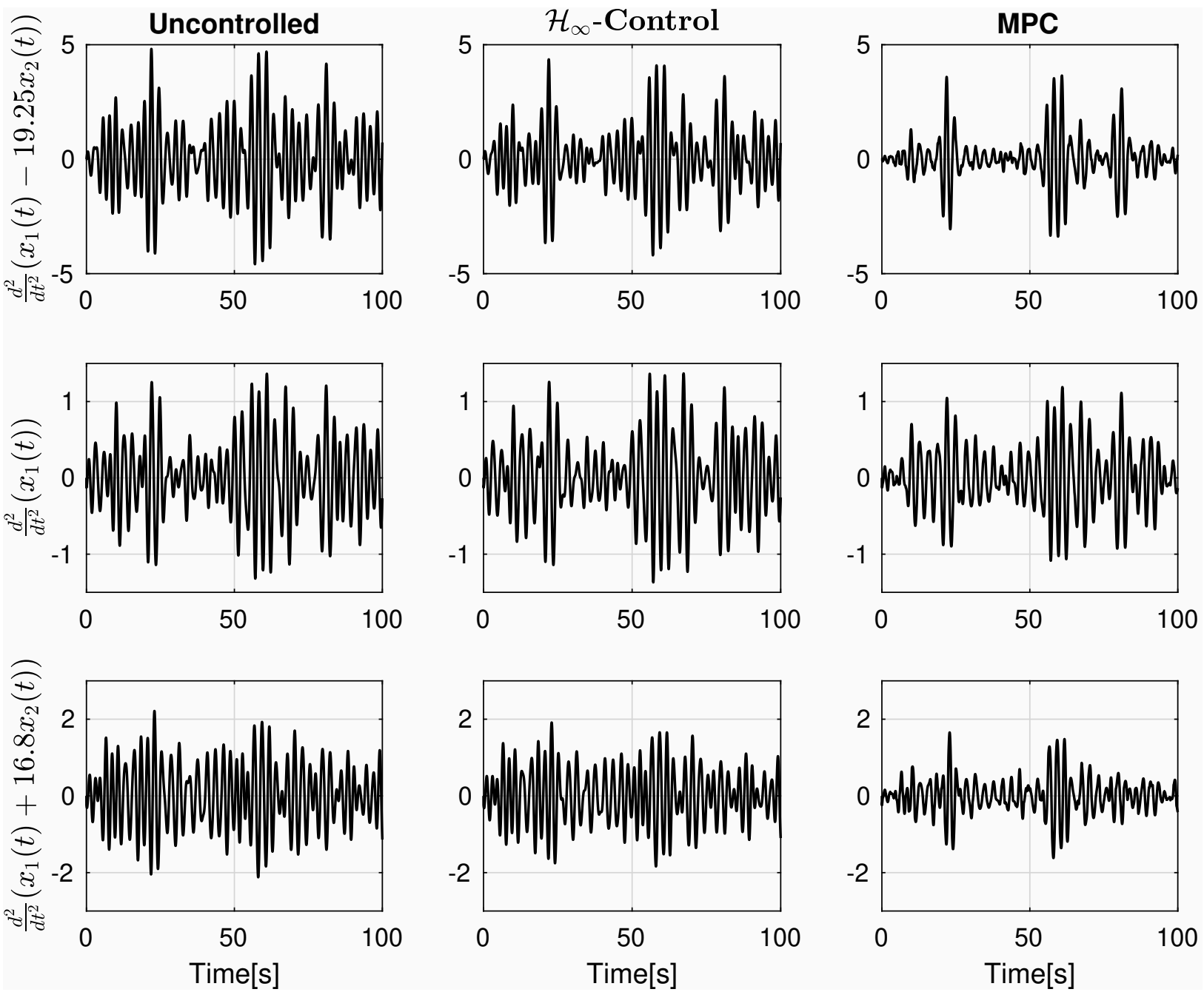

Figure 20: Time histories of uncontrolled and controlled accelerations at $F_{n}=0.50\left(H_{s}=1.00 \mathrm{~m}\right)$.

Table 11: Percentage MSI values for uncontrolled case (NC) and model predictive controlled case (C) at different significant wave heights $\left(H_{s}\right)\left(F_{n}=0.50\right)$.

\begin{tabular}{lccc}
\hline \hline & MSI $H_{s}=0.70$ & MSI $H_{s}=0.88$ & MSI $H_{s}=1.00$ \\
& $\mathrm{NC}[\%]-\mathrm{C}[\%]$ & $\mathrm{NC}[\%]-\mathrm{C}[\%]$ & $\mathrm{NC}[\%]-\mathrm{C}[\%]$ \\
\hline Bow Vertical Acceleration & $1.32-0.0002$ & $6.91-0.27$ & $8.34-0.60$ \\
Stern Vertical Acceleration & $0.069-0.0000156$ & $0.98-0.02$ & $1.91-0.07$ \\
CoG Acceleration & $0.037-0.0002$ & $0.20-0.03$ & $0.85-0.37$ \\
\hline
\end{tabular}

275 and velocity. The results showed that MPC results present better performance than $\mathcal{H}_{\infty}$ discrete time state feedback controller since MPC has advantages in a wave attenuation problem with its preview capability of excitations and handling the constraints. Thanks to the designed MPC discrete based controller, vertical 

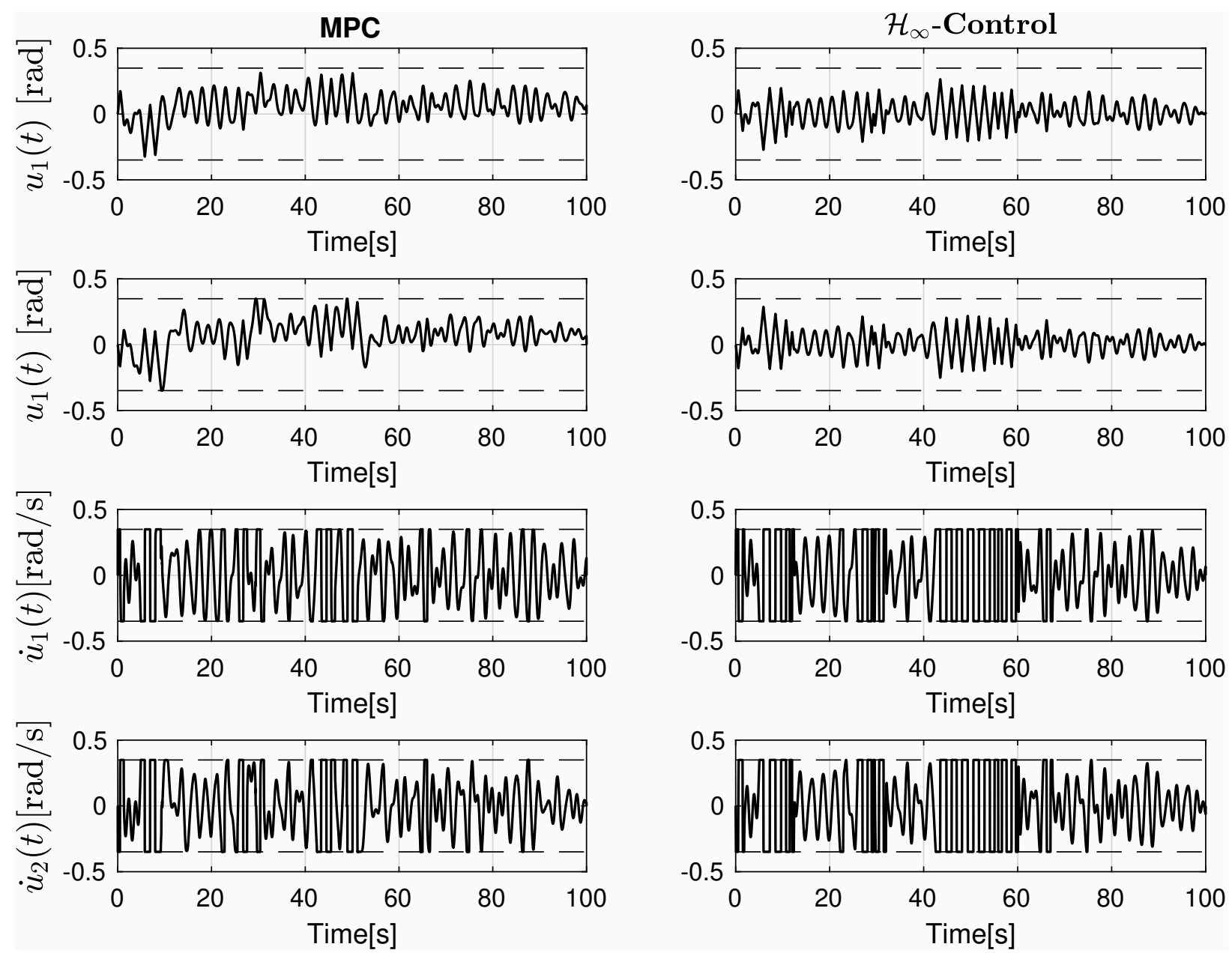

Figure 21: Time Histories of bow and stern foil angles and rates $\left(H_{s}=0.70 \mathrm{~m}\right)$ at $F_{n}=0.40$. Here, dotted horizontal lines represent the physical bounds on the corresponding actuator signal.

accelerations in the bow, centre and stern locations of the ship were reduced by approximately $75 \%, 64 \%$ and $49 \%$, respectively with a significant reduction of pitch motion in $H_{s}=0.70$ metres at $\left.F_{n}=0.40\right)$. On the other hand, vertical accelerations in the bow, centre and stern locations of the ship were reduced by approximately $82 \%, 77 \%$ and $56 \%$, respectively in $H_{s}=0.70$ metres at $F_{n}=0.50$ ). As the magnitude of the sea state increases to $H_{s}=0.88$ and 1 metres, the reduction rates were decreased to the level of $45 \%$ due to the excitation terms are remarkably increased for both $F_{n}$. In other words, as the foils have strict limitations on their opening angles and rotation rates, improvements in the performance of controllers were decreased. It was noted that, in contrast to pitch motion, the same motion reduction was not observed for the heave motion and midship acceleration due to the phase differences between the pitch and heave motions.

In contrast to the general belief in the community which states that active motion attenuation can only be effective on high-speed ships, the results of this study showed that MPC could still provide remarkable 

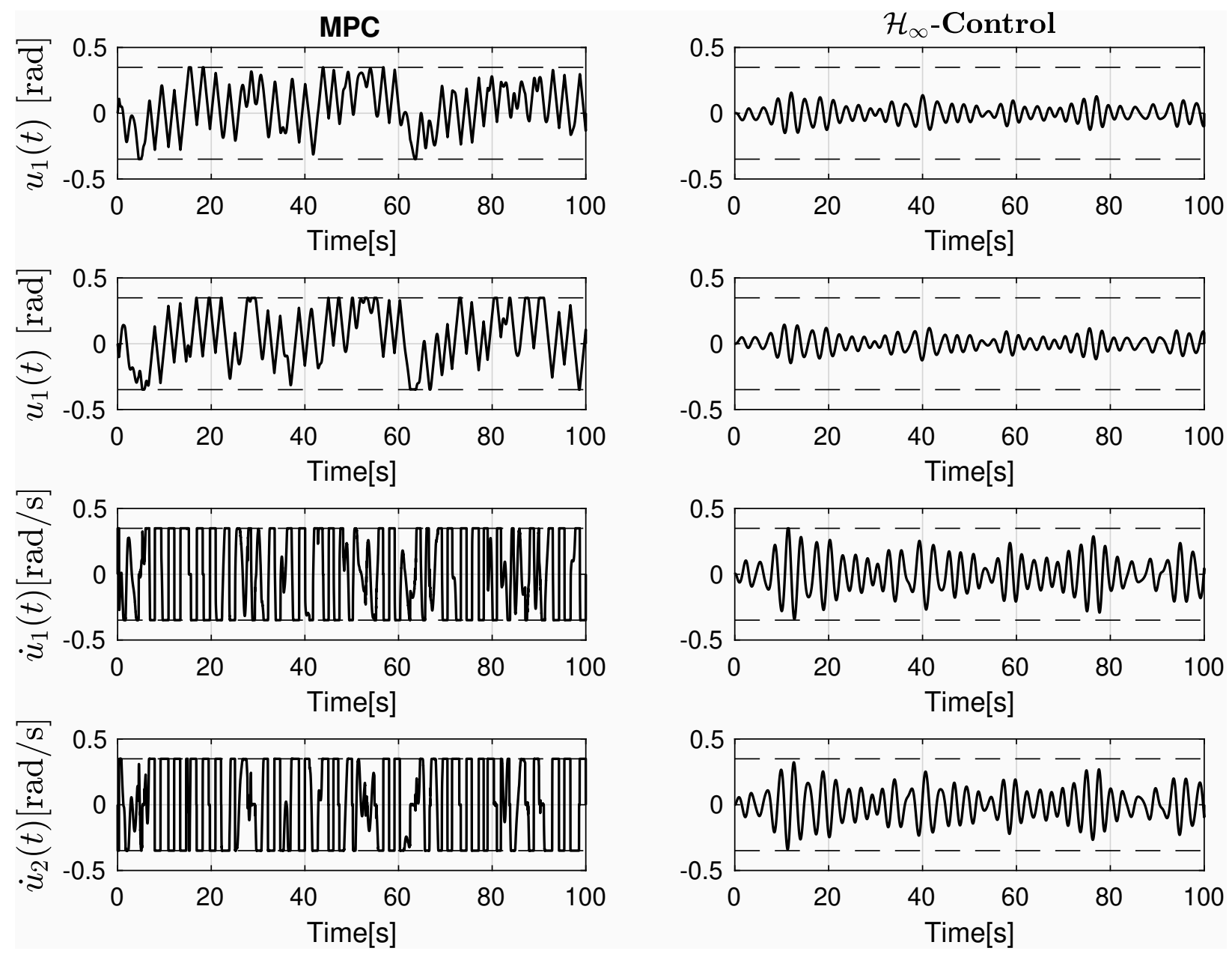

Figure 22: Time histories of bow and stern foil angles and rates $\left(H_{s}=0.88 \mathrm{~m}\right)$ at $F_{n}=0.40$. Here, dotted horizontal lines represent the physical bounds on the corresponding actuator signal.

performance even on moderate-speed vessels having strict actuator amplitude and rate limits. The developed

\section{Acknowledgements}

The second author was supported by ASELSAN Graduate Scholarship for Turkish Academicians. 

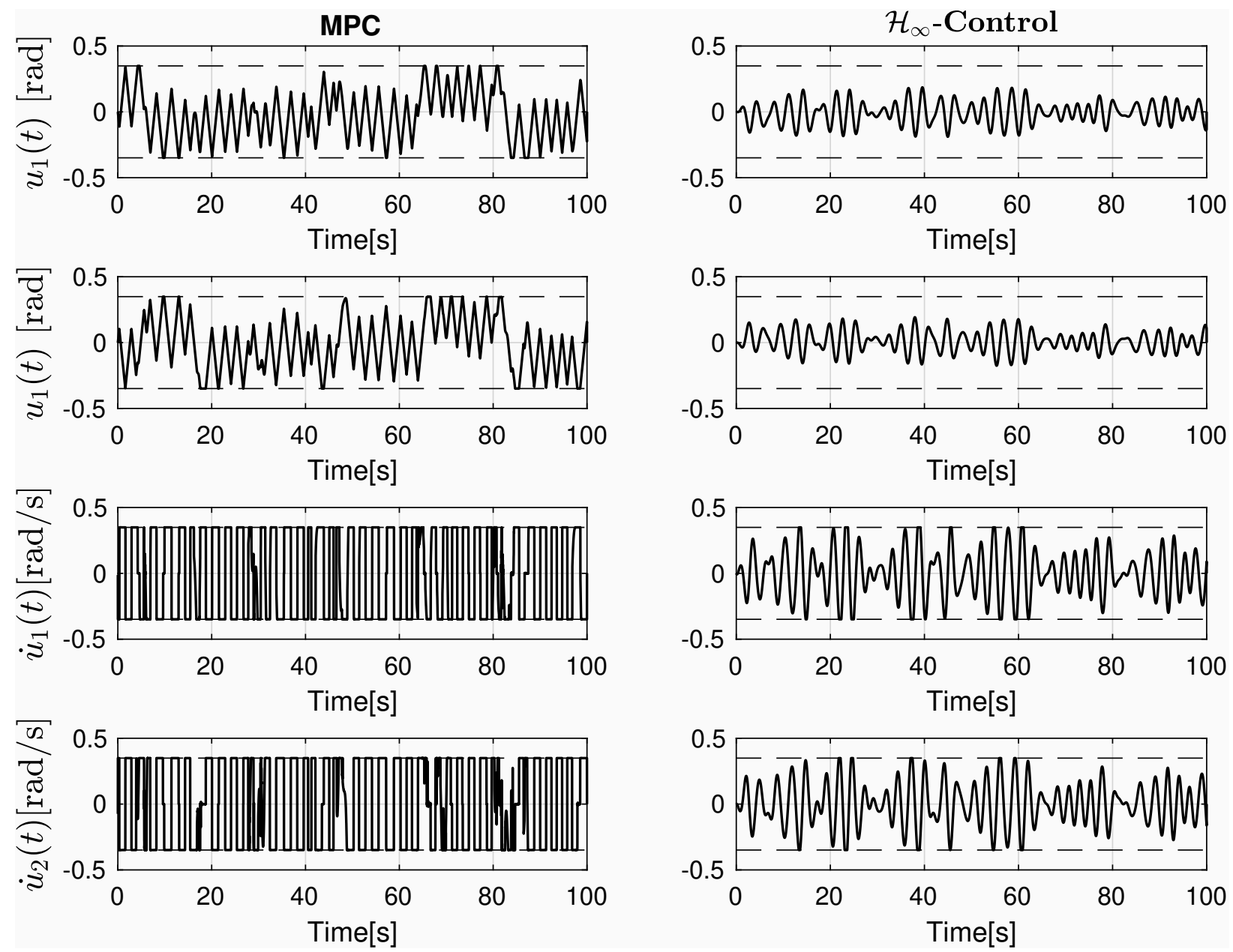

Figure 23: Time histories of bow and stern foil angles and rates $\left(H_{s}=1.00 \mathrm{~m}\right)$ at $F_{n}=0.40$. Here, dotted horizontal lines represent the physical bounds on the corresponding actuator signal.

\section{References}

[1] J. F. O'Hanlon, M. E. McCauley, Motion sickness incidence as a function of acceleration of vertical sinusoidal motion, Aerospace Medicine 45 (1974) 366-369.

300 [2] E. V. Lewis, Ship speeds in irregular seas, Trans. SNAME (1955) 134-202.

[3] M. A. Abkowitz, The effect of anti-pitching fins on ship motions, Trans. SNAME (1959) 210-252.

[4] J. Avis, Use of anti-pitch hydrofoil to reduce added resistance of a yacht in waves, Marine Technology and SNAME News 28 (1991) 14-22.

[5] T. Perez, Course Keeping and Roll Stabilisation Using Rudder and Fins, Springer, 2005.

305 [6] L. Huang, Y. Han, W. Duan, Y. Chen, S. Ma, Numerical and experimental studies on a predictive control approach for pitch stabilization in heading waves, Ocean Engineering (2018) 388-400.

[7] S. Esteban, J. M. Giron-Sierra, B. A. Toro, J. M. De la Cruz, Development of a control-oriented model of the vertical motions of a fast ferry, Journal of Ship Research 48 (2004) 218-230.

[8] J. M. Giron-Sierra, S. Esteban, B. Andres, J. M. S. Martinez, J. Riola, Experimental study of controlled flaps and t-foil 

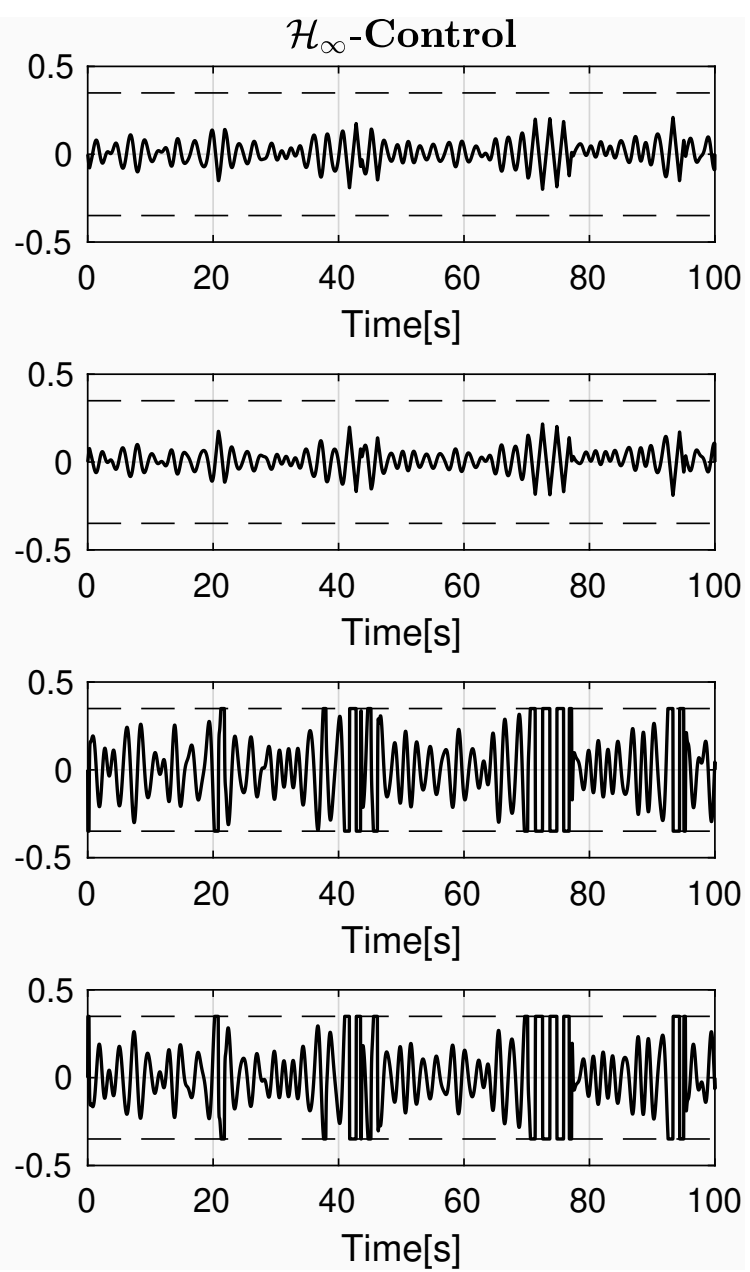

Figure 24: Time Histories of bow and stern foil angles and rates $\left(H_{s}=0.70 \mathrm{~m}\right)$ at $F_{n}=0.50$. Here, dotted horizontal lines represent the physical bounds on the corresponding actuator signal.

for comfort improvement of a fast ferry, in: Control Application in Marine System, IFAC Conference, 2001.

[9] R. López, M. Santos, O. Polo, S. Esteban, Experimenting a fuzzy controller on a fast ferry, in: Control Applications, 2002. Proceedings of the 2002 International Conference on, Vol. 2, IEEE, 2002, pp. 1082-1087.

[10] M. Ticherfatine, Z. Quidan, Model free approach based on intelligent pd controller for vertical motion reduction in fast ferries, Turkish Journal of Electrical Engineering and Computer Sciences 6 (2018) 393-406.

11] M. Ticherfatine, Z. Quidan, Fast ferry smoothing motion via intelligent pd controller, Journal of Marine Science And Applications 17 (2018) 273-279.

[12] S. Zhang, M. Sun, L. Liang, S. Li, H-infinity output feedback control method for the t-foil of the wave piercing catamaran, in: Intelligent Control and Automation (WCICA), 2014 11th World Congress on, IEEE, 2014, pp. 4338-4343.

[13] L. Huang, Y. Han, W. Duan, Y. Zheng, S. Ma, Ship pitch-roll stabilization by active fins using a controller based on onboard hydrodynamic prediction, Ocean Engineering (2018) 212-227.

[14] F. Cakici, H. Yazici, A. D. Alkan, Optimal control design for reducing vertical acceleration of a motor yacht form, Ocean Engineering 169 (2018) 636-650.

[15] F. Borrelli, A. Bemporad, M. Morari, Predictive control for linear and hybrid systems, Cambridge University Press, 2017. 

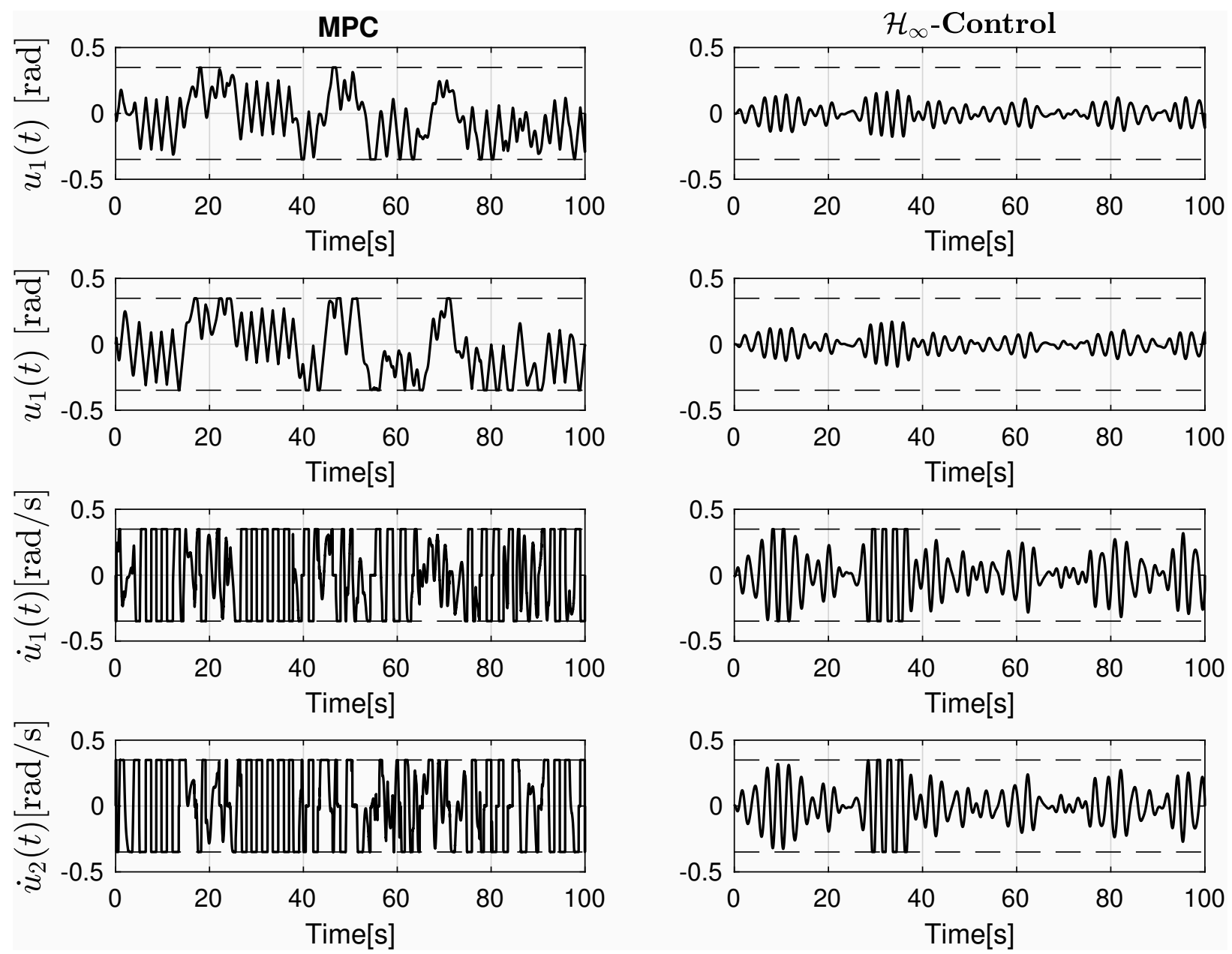

Figure 25: Time histories of bow and stern foil angles and rates $\left(H_{s}=0.88 \mathrm{~m}\right)$ at $F_{n}=0.50$. Here, dotted horizontal lines represent the physical bounds on the corresponding actuator signal.

[16] I. Kose, F. Jabbari, Control of systems with actuator amplitude and rate constraints, Vol. 6, American Control Conference, USA, 2001, pp. 4914-4919.

[17] W. Cummins, The impulse response function and ship motions, Schiffstechnikl 9 (1962) 101-109.

[18] S. Bochner, K. Chandrasekharan, et al., Fourier transforms, Princeton University Press, 1949.

[19] M. Alves, M. Vicente, A. Sarmento, M. Gurerinel, Implementation and verification of a time domain model to simulate the dynamics of owc's, in: 9th European Wave and Tidal Energy Conference, 2011.

330 [20] J. F. Hauer, C. J. Demeure, L. L. Scharf, Initial results in prony analysis of power system response signals, IEEE Transactions on Power Systems 5 (1) (1990) 80-89.

[21] W. J. Pierson Jr, L. Moskowitz, A proposed spectral form for fully developed wind seas based on the similarity theory of SA Kitaigorodskii, Journal of geophysical research 69 (24) (1964) 5181-5190.

[22] M. St Denis, W. Pierson, On the motions of ships in confused seas, Trans. Soc. Nav. Archit. Mar. Eng. 61 (1953) $280-354$.

335 [23] J. H. Kim, Y. H. Kim, Motion control of a cruise ship by using active stabilizing fins, Proc. IME M J. Eng. Marit. Environ 225 (4) (2011) 311-324.

[24] K. Hu, D. Yong, W. Hongwei, High-speed catamaran's longitudinal motion attenuation with active hydrofoils, Polish 

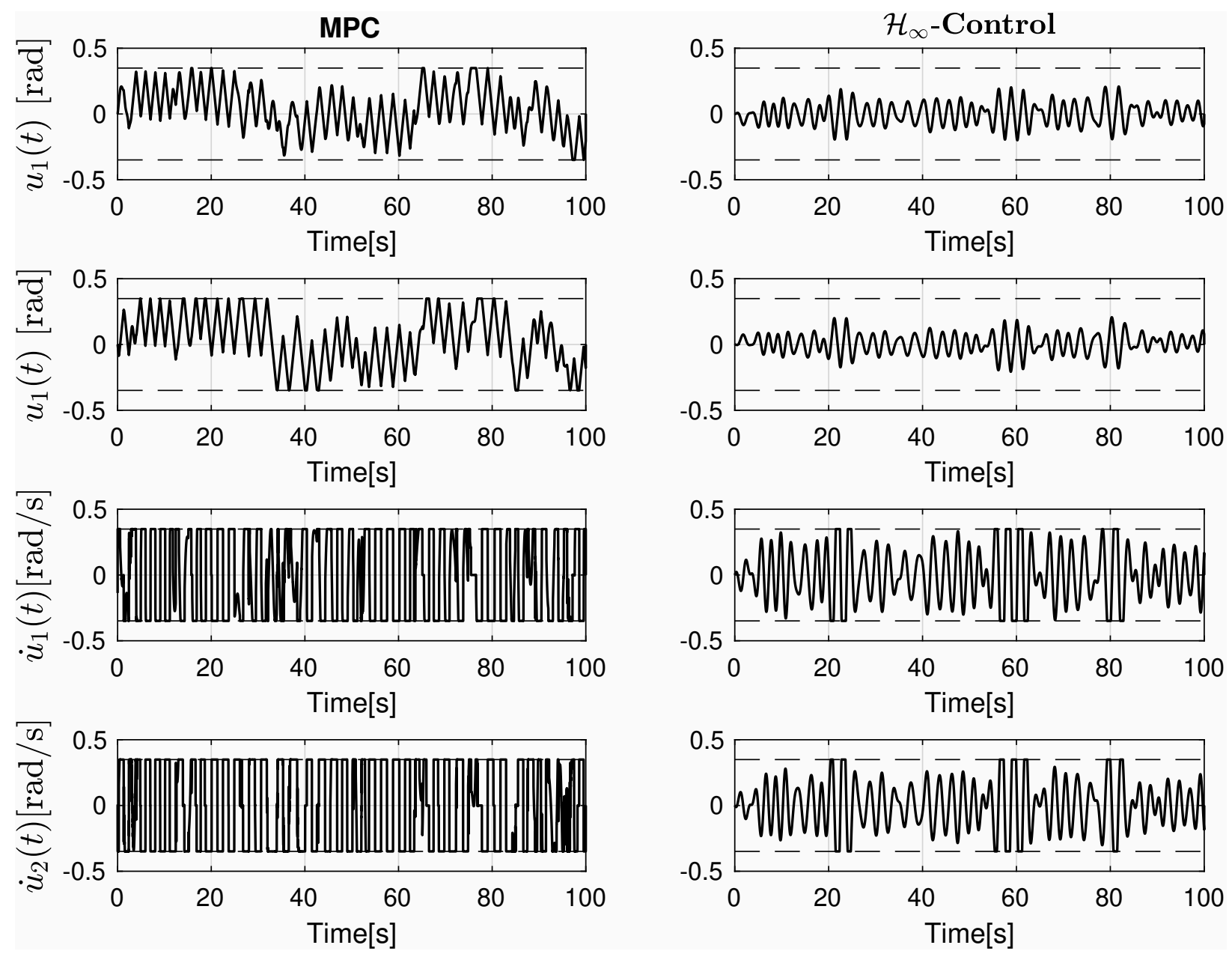

Figure 26: Time histories of bow and stern foil angles and rates $\left(H_{s}=1.00 \mathrm{~m}\right) F_{n}=0.50$. Here, dotted horizontal lines represent the physical bounds on the corresponding actuator signal.

Maritime Research 25 (2018) 56-61.

[25] M. Sadeghi Reineh, S. S. Kia, F. Jabbari, New anti-windup structure for magnitude and rate limited inputs and peakbounded disturbances, Automatica 97 (2018-11) 301,305.

[26] S. Boyd, L. El Ghaoui, E. Feron, V. Balakrishnan, Linear Matrix Inequalities in System and Control Theory, SIAM studies in applied mathematics: 15, 1994.

[27] J. Löfberg, Yalmip : A toolbox for modeling and optimization in matlab, in: In Proceedings of the CACSD Conference, Taipei, Taiwan, 2004.

345 [28] A. Lloyd, Seakeeping: Ship Behaviour in Rough Weather, Ellis Horwood series in marine technology, A.R.J.M. Lloyd, 1998. 
LaTeX Source for the revised paper
Click here to download LaTeX Sourc

LaTeX Source for the revised paper
Click here to download LaTeX Source Files: revised paper_Ocean Engineering.rar (1)

Click here to download LaTeX Source Files: revised paper_Ocean Engineering.rar

Click here to download LaTeX Source Files: revised paper_Ocean Engineering.rar

lick here to download LaTeX Source Files: revised paper_Ocean Engineering.rar

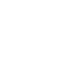

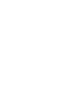

lick here to download LaTeX Source Files: revised paper_Ocean Engineering.rar

Click here to download LaTeX Source Files: revised paper_Ocean Engineering.rar

lick here to download LaTeX Source Files: revised paper_Ocean Engineering.rar 更

lick here to download LaTeX Source Files: revised paper_Ocean Engineering.rar lick here to download LaTeX Source Files: revised paper_Ocean Engineering.rar

lick here to download LaTeX Source Files: revised paper_Ocean Engineering.rar

lick here to download LaTeX Source Files: revised paper_Ocean Engineering.rar lick here to download LaTeX Source Files: revised paper_Ocean Engineering.rar lick here to download LaTeX Source Files: revised paper_Ocean Engineering.rar lick here to download LaTeX Source Files: revised paper_Ocean Engineering.rar

Click here to download LaTeX Source Files: revised paper_ocean Engineering.rar lick here to download LaTeX Source Files: revised paper_Ocean Engineering.rar lick here to download LaTeX Source Files: revised paper_Ocean Engineering.rar

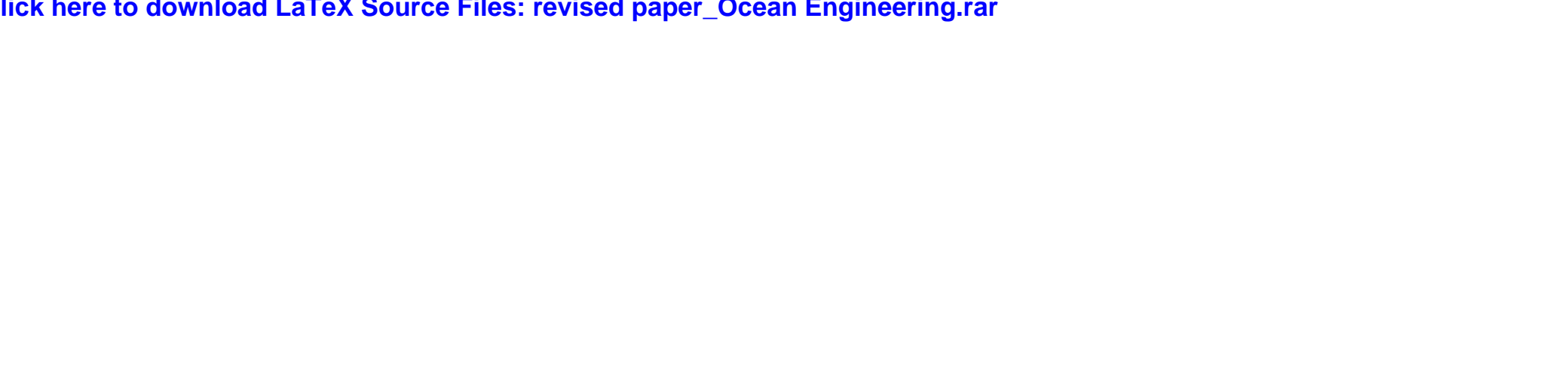

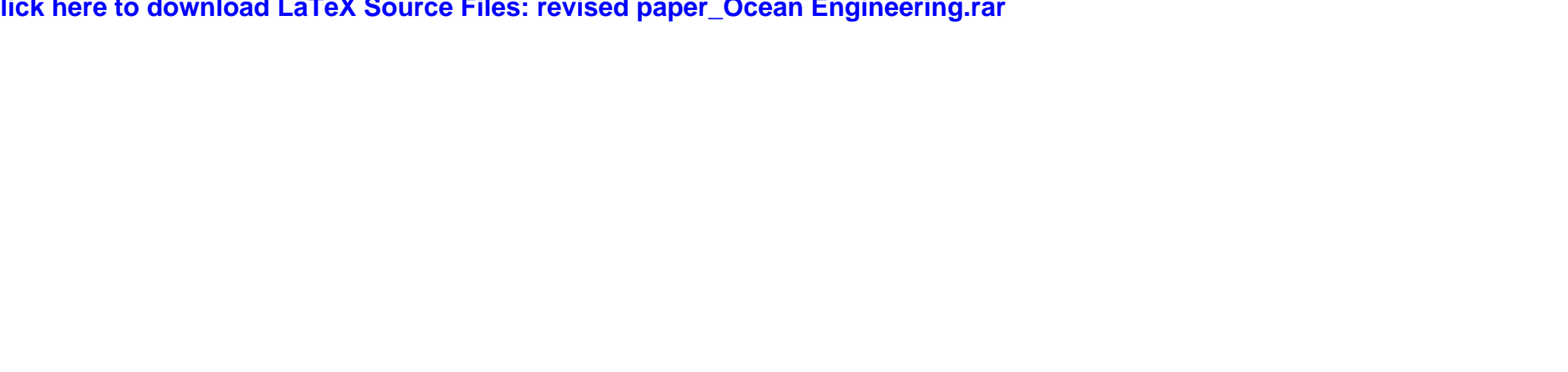

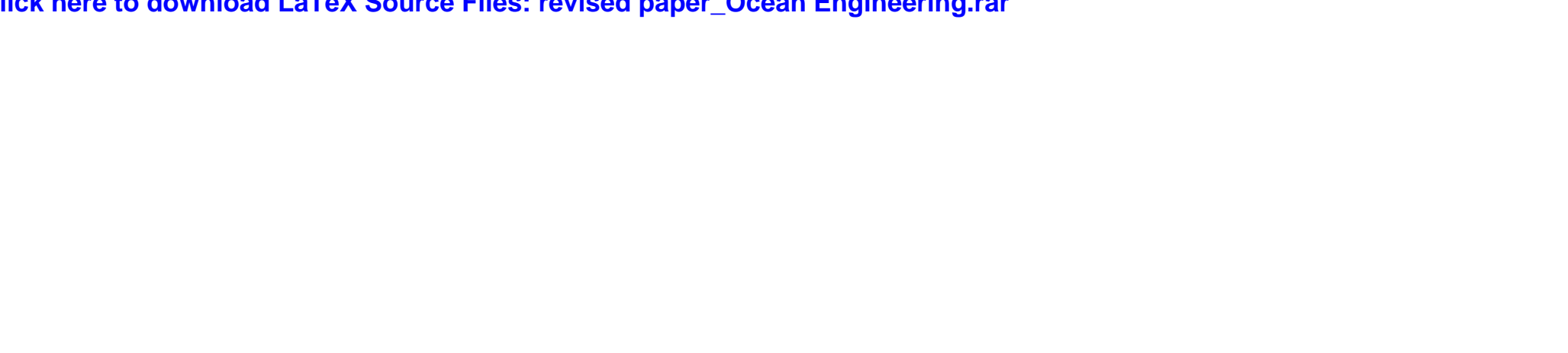

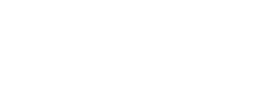
列

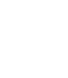

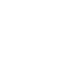

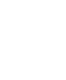

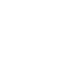

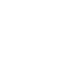
更 